\title{
Ecología de la restauración en México: estado actual y perspectivas
}

\author{
Restoration ecology in Mexico: state of the art and perspectives \\ Fabiola López-Barrera ${ }^{\mathrm{a}, *}$, Cristina Martínez-Garza ${ }^{\mathrm{b}}$ y Eliane Ceccon ${ }^{\mathrm{c}}$ \\ ${ }^{a}$ Red de Ecología Funcional, Instituto de Ecología, A.C., Carretera Antigua a Coatepec Núm. 351, El Haya, 91070, Xalapa, Veracruz, México \\ ${ }^{\mathrm{b}}$ Centro de Investigación en Biodiversidad y Conservación, Universidad Autónoma del Estado de Morelos, Avenida Universidad 1001, Colonia Chamilpa, 62209, \\ Cuernavaca, Morelos, México \\ ${ }^{c}$ Centro Regional de Investigaciones Multidisciplinarias, Universidad Nacional Autónoma de México, Av. Universidad s/n, Circuito 2, Colonia Chamilpa, 62210, \\ Cuernavaca, Morelos, México
}

Recibido el 2 de marzo de 2016; aceptado el 13 de septiembre de 2016

Disponible en Internet el 8 de noviembre de 2017

\section{Resumen}

La ecología de la restauración es una disciplina científica que a partir de la teoría ecológica desarrolla principios para guiar la práctica de la restauración de los ecosistemas. El objetivo de esta revisión es exponer una síntesis de la situación actual y las perspectivas de la ecología de la restauración en México, así como los retos de la investigación ante el escenario ambiental de nuestro país. Se realizó una revisión de diversos indicadores, como publicaciones, congresos, instituciones, investigadores y oferta de formación de recursos humanos. La revisión arrojó un total de 206 artículos (1995-2016), de los cuales el 78\% correspondió a artículos de investigación, el 18\% a artículos de opinión y propuestas metodológicas y el $4 \%$ fueron metaanálisis. En el $82 \%$ de las publicaciones, el primer autor tuvo su adscripción en una institución mexicana, y de ellos el $57 \%$ correspondió a hombres y el $43 \%$ a mujeres. Los estudios sobre ecosistemas forestales representaron el $71 \%$, abordando principalmente la recuperación de especies de árboles y arbustos. La evaluación mostró que algunos indicadores tuvieron incrementos pero otros se mantuvieron constantes. También se hizo evidente que la oferta académica no ha sido suficiente, y se discute la necesidad de una política científica nacional de restauración así como fuentes de financiamiento a largo plazo.

(C) 2017 Universidad Nacional Autónoma de México, Instituto de Biología. Este es un artículo Open Access bajo la licencia CC BY-NC-ND

(http://creativecommons.org/licenses/by-nc-nd/4.0/).

Palabras clave: Restauración de ecosistemas; Formación de capacidades; Restauración ecológica

\begin{abstract}
Restoration ecology is a scientific discipline which develops principles to guide the restoration of ecosystems based in ecological theory. The objective of this review is to synthetize the current situation and the perspective of the restoration ecology in Mexico and the challenge of this area of research given the environmental conditions in our country. A review of different indicators as scientific publications, meetings, institutions, researchers and the offer of academic programs was carried out. This review resulted in a total of 206 articles from which $78 \%$ were research articles (1995-2016), 18\% opinions or methodological proposals and $4 \%$ were meta-analyses. In $82 \%$ of the publications, the first authors were working at a Mexican institution and from them, $57 \%$ were men and $43 \%$ women. The studies on forest ecosystems represented $71 \%$ and the principal topic was the recovery of woody species. The evaluation showed that some indicators increased whereas other stayed constant. The results showed that the academic offer was not enough; the need of a national scientific policy of restoration and long-term sources of funding is discussed. (C) 2017 Universidad Nacional Autónoma de México, Instituto de Biología. This is an open access article under the CC BY-NC-ND license (http://creativecommons.org/licenses/by-nc-nd/4.0/).
\end{abstract}

Keywords: Ecosystem restoration; Capacity building; Restoration ecology

\footnotetext{
* Autor para correspondencia.

Correo electrónico: fabiola.lopez@inecol.mx (F. López-Barrera).

La revisión por pares es responsabilidad de la Universidad Nacional Autónoma de México.
} 


\section{Introducción}

México cuenta con una alta heterogeneidad ambiental que propicia una gran diversidad biológica y un capital natural considerable. El país se encuentra entre las 5 primeras naciones con mayor riqueza biológica y cultural; sin embargo, dicho capital natural ha sufrido un alarmante deterioro por factores de cambio directos e indirectos, así como por las interacciones de estos (Challenger, Dirzo, López, Mendoza y Lira-Noriega, 2009; Domínguez et al., 2009; Sarukhán et al., 2015). Entre los principales factores de cambio directos se encuentra la conversión de los hábitats naturales a otros usos de suelo y su consecuente fragmentación y degradación; por ejemplo, ya para 1993 solo existía el $54 \%$ de la cobertura original de la vegetación natural del país, llegando en 2002 a ocupar solo el 38\% del territorio, y de esa cobertura, alrededor del 50\% se consideraba vegetación degradada (Challenger et al., 2009).

En una reciente evaluación forestal internacional se reconoció que en los últimos 5 años (2010-2015) la tasa de deforestación en México disminuyó ( $-0.1 \%$ /año) con respecto al período de 1990 a 2000 (-0.3\%/año; FAO, 2015a, 2015b); sin embargo, nuestro país continúa perdiendo la poca superficie de bosque primario que aún le queda $(-0.7 \% / a n ̃ o)$ y se ha registrado una intensificación reciente en la degradación de los bosques debido a causas antropogénicas directas (FAO, 2015a). Por ejemplo, en un muestreo del inventario forestal nacional se estimó que del 70 al $80 \%$ de las parcelas evaluadas tenían uno o más indicadores de disturbio, como daño por fuego, presencia de tocones, pastoreo o extracción selectiva, entre otros (Morales-Barquero et al., 2014). Por otra parte, aunque existen menos indicadores de la degradación de ecosistemas costeros y marinos, en México se han documentado para los manglares tasas de pérdida de cobertura anual que van del $-0.08 \%$ al $-3.94 \%$ entre 1970 y 2005 (RodríguezZúñiga et al., 2013). En cuanto a las dunas costeras, se ha estimado que en México existen aproximadamente 800,000 ha (el $1 \%$ de la superficie del país), y cerca del 50\% han sido transformadas para uso agropecuario o han sido urbanizadas (Martínez et al., 2014). Igual de desalentador es el caso de los humedales, ya que se estima que el país ha perdido el $62 \%$ de los humedales, que son ecosistemas prioritarios por brindar múltiples servicios ambientales (Landgrave y Moreno-Casasola, 2012).

Otro factor de cambio es la invasión de especies exóticas; actualmente en el país residen al menos 46 de las 100 especies invasoras más dañinas del mundo y afectan todos los ecosistemas (Aguirre-Muñoz et al., 2009). En México, un total de 127 especies de plantas y animales ya se han extinguido o han sido extirpadas (Baena y Halffter, 2008) y más de 2,000 se encuentran registradas en diferentes grados de amenaza en la Norma Oficial Mexicana (NOM-059-SEMARNAT-2010). Otro aspecto preocupante es la pérdida o el decremento de poblaciones de animales y plantas; estas extinciones locales no se reflejan en los indicadores nacionales de degradación y constituyen una pérdida del acervo genético local (Sarukhán et al., 2015). Entre los factores indirectos, el cambio climático se ha convertido en una amenaza cercana para la biodiversidad. Por ejemplo, México alberga 373 especies de anfibios, y contamos con el mayor número de endemismos en el mundo para este grupo (>65\%); sin embargo, siendo un grupo biológico con requerimientos microambientales muy específicos, se estima que bajo escenarios de cambio climático se darán extinciones intensas de este grupo, particularmente en el período de 2020 a 2050 (OchoaOchoa, Rodríguez, Mora, Flores-Villela y Whittaker, 2012).

La pérdida del capital natural también se refleja en un decremento en la calidad de vida y la seguridad alimentaria. Por ejemplo, se estima que al menos la cuarta parte de los mexicanos tiene un acceso deficiente a la alimentación (Urquía-Fernández, 2014). Los problemas de producción de alimentos se relacionan con los suelos degradados o improductivos que prevalecen en el $45 \%$ del territorio mexicano (Conabio, 2009) y procesos de desertificación en el 14\% del suelo (Bollo-Manent, HernándezSantana y Méndez-Linares, 2014). En un informe reciente de la Comisión Nacional del Agua se encontró que, en algunas regiones hidrológicas del país, hasta el 55\% de sus cuerpos de agua superficiales se encontraban contaminados por materia orgánica, y a nivel nacional el $16.3 \%$ de los acuíferos se encontraban sobreexplotados (CNA, 2013).

Esta grave alteración en los ecosistemas de México y sus efectos en la sociedad resaltan la urgencia de desarrollar estrategias de conservación y restauración. La restauración puede prevenir y revertir la pérdida de la diversidad biológica así como promover la recuperación de los servicios ecosistémicos (SER, 2004). Ante este escenario, es inaplazable establecer una agenda de investigación con temas prioritarios con el fin de determinar el umbral crítico donde los cambios en la estructura y función de los ecosistemas ya han sido o serán irreversibles (Cantarello et al., 2011). La ecología de la restauración es una disciplina científica que, a partir de la teoría ecológica, desarrolla principios para guiar la práctica de la restauración (fig. 1); la restauración ecológica se define como el proceso de asistir a la recuperación de un ecosistema que ha sido dañado, degradado o destruido (SER, 2004). Desde la conceptualización de la ecología de la restauración por Bradshaw (1987) surgió una oportunidad única de experimentación para probar hipótesis sobre los procesos de recuperación y resiliencia de la estructura y función de los ecosistemas.

La ecología de la restauración se ha convertido en una disciplina científica que ya no solo observa y documenta, sino también explica y predice (Howe y Martínez-Garza, 2014). Esto se ve reflejado en el crecimiento de revistas indizadas especializadas sobre el tema, publicaciones, programas de investigación y de formación de recursos humanos en México y el mundo (Martínez y López-Barrera, 2008). Por ejemplo, en solo 15 años (1990-2004) los artículos de restauración llegaron a representar el 5\% de los artículos de ecología a nivel mundial (Young, Petersen y Clary, 2005). Actualmente, en la base JCRWoS (www.webofknowledge.com) se pueden encontrar más de 3,700 artículos de ecología que en su título mencionan la restauración y/o la rehabilitación. En este momento, Estados Unidos es la potencia en este campo y China se encuentra en segundo lugar, mientras que México ocupa el lugar número 13 (fig. 2).

Considerando los escenarios ambientales en México y el auge de la ecología de la restauración en el mundo, el objetivo de esta revisión es exponer una síntesis de la situación actual y las 


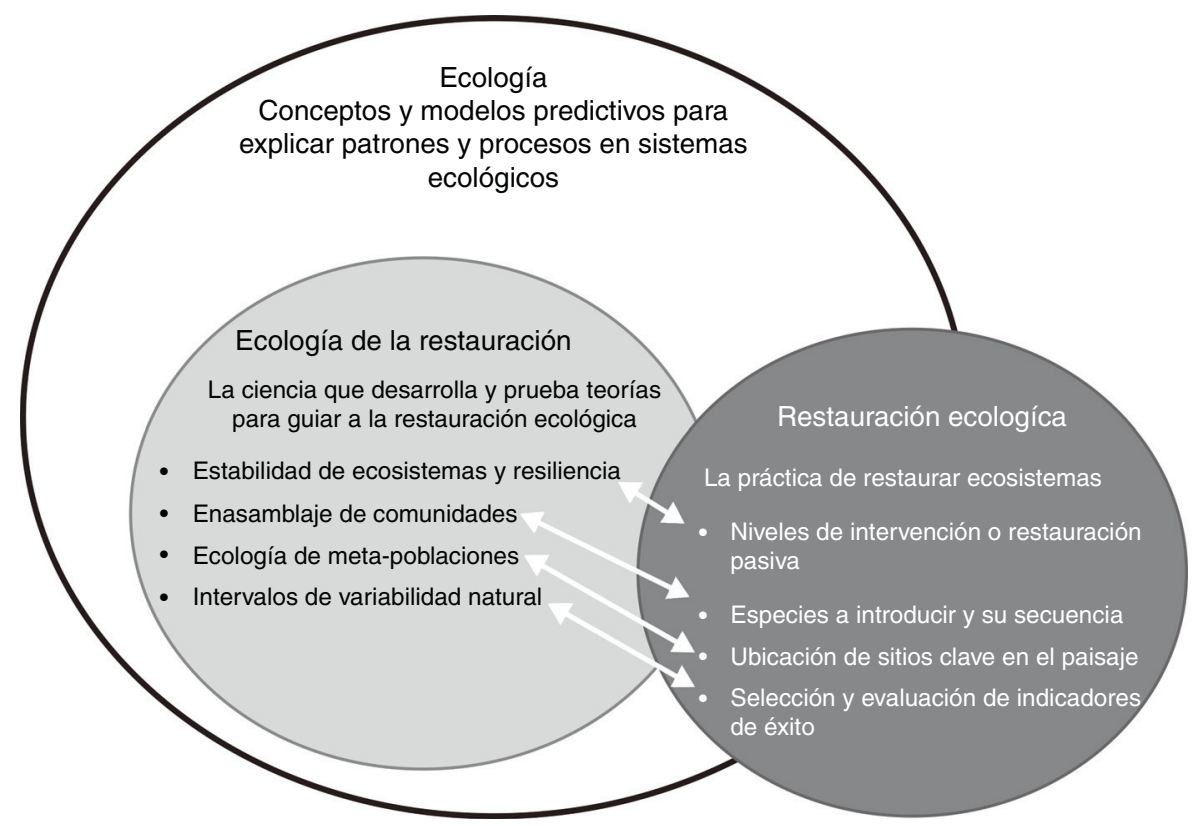

Figura 1. Relación entre la ecología, la ecología de la restauración y la práctica de la restauración ecológica. Modificado de Palmer (2009).

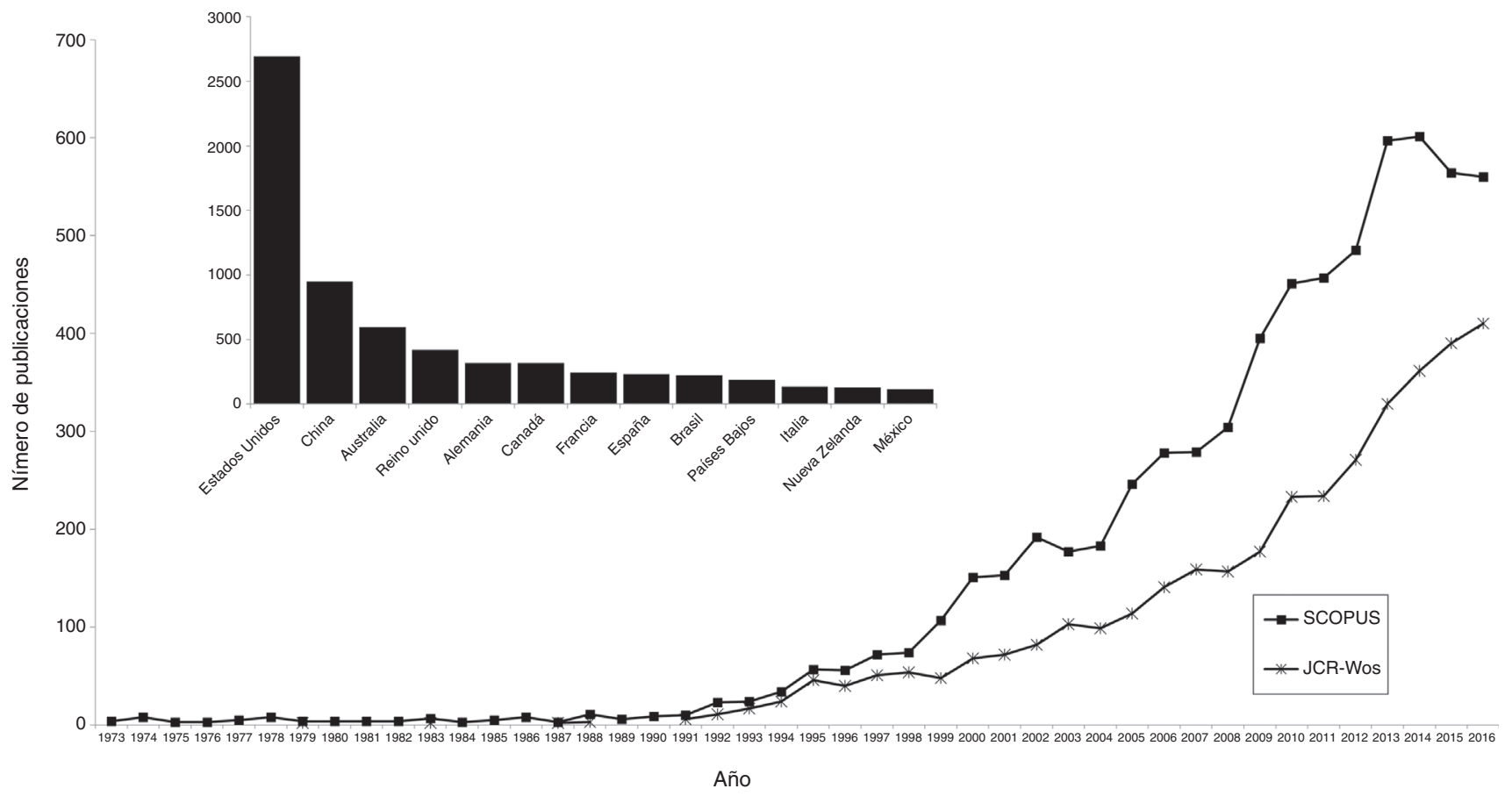

Figura 2. Crecimiento en el número de publicaciones según la base de datos SCOPUS (consulta: título [restor* or rehab*] y título+resumen+palabras clave [ecolo*]) y en la base JCR-WoS (consulta: títutlo [restor* or rehab*] y tema [ecolo*]). Se muestran los países con más publicaciones acumuladas según la base de datos de SCOPUS (1973-2016).

perspectivas de la ecología de la restauración en nuestro país. Nuestra meta es exponer algunos de los aportes básicos y aplicados para responder las siguientes preguntas: ¿cuáles son las bases sobre las que se desarrolló la ecología de la restauración en México como ciencia?, ¿cuál es la visión actual de la ecología de la restauración en México?, ¿hacia dónde se dirige?, y ¿cuáles son los retos, nuevas tendencias y perspectivas a futuro?

\section{Métodos}

Con el fin de conocer el número y el tipo de investigación que se realiza sobre ecología de la restauración por autores adscritos a instituciones mexicanas, en enero de 2017 se realizó una consulta solo de artículos en la base de datos SCOPUS (www.scopus.com) con los siguientes criterios de inclusión: restor* or rehab* en el título and ecolo* en el 
título, resumen y/o palabras clave. También se consultó la base JCR-WoS (www.webofknowledge.com), incluyendo en la búsqueda restor* or rehab* en el título and ecolo* en el tema. Después, en ambos casos se filtraron aquellos artículos que presentaron alguna institución mexicana, como la adscripción de alguno de los autores. Ambas bases se conjuntaron y se eliminaron las referencias repetidas. Posteriormente se hizo una búsqueda ampliada usando las bases de Google académico (https://scholar.google.es/) y la Red de Revistas Científicas de América Latina y el Caribe, España y Portugal (www.redalyc.org) usando las palabras restau*, rehab* y México en la búsqueda. Adicionalmente, se consultó la lista bibliográfica de una compilación de Bonfil, Fernández y Fernández y González-Espinosa (2015) para completar la base. Cabe mencionar que dicha compilación cuenta con 322 referencias reportadas como estudios y artículos de difusión relacionados con la restauración ecológica en México. Los criterios del presente estudio fueron más acotados y estrictos, específicamente se buscó que los estudios pertenecieran a la ecología de la restauración y que explícitamente abordaran preguntas, opiniones o revisiones sobre la restauración de ecosistemas por autores con adscripción en instituciones mexicanas. La base final de referencias fue clasificada de acuerdo al tipo de aportación (investigación, metaanálisis, revisión, opinión o propuesta metodológica), grupos biológicos, ecosistemas, revista, instituciones académicas, género del primer autor, género y posición del autor mexicano y número total de autores de las publicaciones.

Con el fin de estimar el impacto de la ecología de la restauración en la práctica se compilaron los manuales y libros generados en México relacionados tanto con la teoría como con la práctica. En enero de 2017 se consultó la base de Google Books (https://books.google.com.mx/) y la sección de novedades editoriales de los boletines electrónicos de la Red Mexicana para la restauración ambiental (www1.inecol.edu.mx/repara/). Con el fin de conocer la aportación de libros y manuales en México con respecto a lo generado en el mundo se hizo una búsqueda ampliada en Google Books usando los términos restauración, rehabilitación y recuperación en español, inglés y portugués. Los libros encontrados fueron depurados para seleccionar los relacionados o vinculados a la ecología de la restauración.

Para conocer el crecimiento de la investigación en torno a la recuperación de los ecosistemas, además del análisis temporal de las publicaciones se contabilizaron los trabajos de este tema que se presentaron como posters o ponencias en simposios o sesiones orales en los congresos de ecología de la Sociedad Científica Mexicana de Ecología, A.C., de 2006 a 2015. Con el fin de estimar qué proporción de investigadores definen su línea de investigación como «restauración ecológica» o «ecología de la restauración», se realizó una revisión de las páginas personales de los investigadores en 4 institutos de investigación ecológica: INECOL, ECOSUR, IE-UNAM e IIES-UNAM.

Para conocer los programas de formación de recursos humanos en México se realizó una búsqueda de la oferta educativa en torno al tema buscando directamente en la red las palabras: cursos, diplomados, maestrías y doctorados junto con las palabras de restauración ecológica y México. La búsqueda se completó utilizando las bases de Universia México (www.universia.net.mx; consultada en julio de 2015) y la de la Red de Ecosistemas y Sustentabilidad (http://redsocioecos.org; consultada en julio de 2015).

Con el fin de documentar las tendencias de la asignación de financiamiento a proyectos en el área de ecología de la restauración se revisaron los resultados de las convocatorias del fondo Ciencia Básica del Consejo Nacional de Ciencia y Tecnología (Conacyt) de 2004 a 2014 y se registraron en una base de datos las propuestas relacionadas con el tema. Adicionalmente, se revisaron los proyectos financiados por el Programa de Apoyo a Proyectos de Investigación e Innovación Tecnológica (PAPIIT) de la UNAM durante el período de 2012 a 2015. Se realizó una búsqueda de la palabra restauración en el título $\mathrm{y}$ en las palabras clave, y los proyectos se concentraron en una base de datos. Para conocer proyectos financiados por la Comisión Nacional para el Conocimiento y Uso de la Biodiversidad (Conabio) se consultaron las propuestas financiadas por el Programa de Compensación y Restauración Ambiental durante el período 1999 al primer semestre de 2015 y se consultó también la revisión realizada por Arriaga (2014).

\section{Resultados}

\section{Fundamentos de la ecología de la restauración en México}

Los principales aportes a la ecología de la restauración por autores mexicanos se han dado en el tema de la recuperación de la vegetación. La base de este conocimiento se encuentra en los estudios florísticos de las selvas conservadas en las décadas de 1960 y 1970 (Gómez-Pompa, 1973; Miranda y Hernández-Xolocotzi, 1963) que permitieron conocer los ahora llamados «ecosistemas de referencia». A finales de la década de 1950 se creó la Comisión de Estudios sobre Dioscóreas (barbasco, Dioscorea composita Hemsl.) con un gran interés de estudiar la estructura y composición de la vegetación secundaria joven, llamada localmente acahuales. A partir de esos estudios se generaron importantes evaluaciones sobre agricultura y conocimiento tradicional (Hernández-Xolocotzi, 1988), sucesión secundaria mediante la evaluación de cronosecuencias (Gómez-Pompa y del Amo, 1985; Gómez-Pompa, del Amo, Vázquez-Yanes y Butanda, 1976; Gómez-Pompa y VázquezYanes, 1981; Purata, 1986a) y la importancia del paisaje en la regeneración de las selvas (Guevara, Purata y van der Maarel, 1986; Purata, 1986b). Estos temas de investigación han sido los pilares de las cuatro líneas actuales de la ecología de la restauración de selvas y bosques: 1) ensambles ecológicos y funcionales para acelerar la sucesión (Álvarez-Aquino y Williams-Linera, 2012; Álvarez-Aquino, Williams-Linera y Newton 2004; Álvarez-Sánchez, Sánchez-Gallén y Guadarrama, 2009; Ceccon, Sánchez y Powers, 2014; De la Rosa-Mera y Monroy-Ata, 2006; Martínez-Garza, Bongers y Poorter, 2013; MartínezGarza, Peña, Ricker, Campos y Howe, 2005; Suárez-Guerrero y Equihua, 2005; Vázquez-Yanes y Batis, 1996); 2) estrategias para la eliminación de especies que detienen la sucesión 
secundaria (Douterlungne, Thomas y Levy-Tacher, 2013; Meli y Dirzo, 2012; Roman-Danobeytia, Levy-Tacher, Aronson, Rodrigues y Castellanos-Albores, 2012); 3) procesos de nucleación y facilitación a nivel de sitio y paisaje (Avendaño-Yáñez, Sánchez-Velásquez, Meave y Pineda-López, 2014; Badano, Pérez y Vergara, 2009, Badano, Samour-Nieva y Flores, 2011; Blanco-García, Sáenz-Romero, Martorell, Alvarado-Sosa y Lindig-Cisneros, 2011; Mendoza-Hernández, Orozco-Segovia, Meave, Valverde y Martínez-Ramos, 2013; Williams-Linera, López-Barrera, Bonilla-Moheno, 2015), y 4) rehabilitación productiva en paisajes con un intenso uso de recursos (Dhillion, Aguilar-Stoen y Camargo-Ricalde, 2004; DíazRodríguez, Blanco-García, Gómez-Romero y Lindig-Cisneros, 2012; Diemont et al., 2006; Douterlungne, Levy-Tacher, Golicher y Danobeytia, 2008; Fuentealba y Martínez-Ramos, 2014; González-Espinosa et al., 2007; Jiménez, Jurado, Aguirre y Estrada, 2005; Murgueitio, Calle, Uribe, Calle y Solorio, 2011; Suárez et al., 2012).

\section{La investigación contemporánea sobre la restauración de ecosistemas}

Dos publicaciones especiales sobre la restauración en México, una en 2007 y otra en 2010, representaron puntos importantes en la historia de investigación contemporánea del tema en nuestro país:

En 2007 se publicó un número especial sobre restauración ecológica en México en el entonces Boletín de la Sociedad Botánica de México (BSBM, ahora Botanical Sciences) como un suplemento. Este número especial incluyó un artículo de presentación, un editorial escrito por José Sarukhán y 7 artículos derivados del simposio «Procesos ecológicos y restauración de comunidades vegetales» organizado por Javier Álvarez-Sánchez y Julia Carabias y que se llevó a cabo dentro de las actividades del XVICongreso Mexicano de Botánica, celebrado en la ciudad de Oaxaca en 2004. Este número especial estuvo a cargo de los organizadores del simposio como editores invitados y del editor en jefe del BSBM, Jorge A. Meave. En el primer artículo de este suplemento se puede apreciar un análisis de las políticas públicas nacionales de las últimas décadas, sus avances y limitaciones (Carabias, Arriaga y Gutiérrez, 2007), mientras que los otros 6 artículos cubrieron diferentes temas de restauración en 5 ecosistemas de México de 4 estados de la República: 2 trabajos fueron realizados en la selva húmeda (en Chiapas: MartínezRamos y García-Orth, 2007, y en Veracruz: Álvarez-Sánchez, Guadarrama-Chávez, Sánchez-Gallén y Olivera, 2007), uno en el bosque de montaña de Chiapas (González-Espinosa et al., 2007), uno en humedales costeros de Veracruz (Flores-Verdugo et al., 2007), uno en el bosque de pino de Michoacán (LindigCisneros, Blanco-García y Sáenz-Romero, 2007) y uno en el matorral xerófilo de Hidalgo (Monroy-Ata, Estévez-Torres, García-Sánchez y Ríos-Gómez, 2007).

En 2010, en la revista Ecological Restoration de la Universidad de Wisconsin, Estados Unidos, se publicó una sección especial de Restauración Ecológica en México, para la cual Roberto Lindig-Cisneros fue el editor invitado. En esta sección se publicaron 12 trabajos de restauración en México: 4 notas de restauración y 8 artículos de investigación. Tres de las notas de restauración tuvieron un nivel de aproximación poblacional: restauración de poblaciones de: 1) Ambystoma mexicanum Shaw (clase Amphibia, orden Caudata, familia Ambystomatidae) en humedales urbanos de la Ciudad de México (Valiente, Tovar, González, Eslava-Sandoval y Zambrano, 2010); 2) Symphoricarpus microphyllus Kunth (Caprifoliaceae) en el Estado de México (Mendoza-Bautista, García-Moreno y Rodríguez-Trejo, 2010) y 3) Buddleja cordata Kunth (Buddlejaceae) en bosques urbanos de la Ciudad de México (Mendoza-Hernández, Orozco-Segovia y Pisanty, 2010). Cuatro de los artículos trataron la restauración de ecosistemas acuáticos y semiacuáticos: humedales en Veracruz (López-Rosas et al., 2010) y la ciudad de México (Von Bertrab y Zambrano, 2010), manglares en Yucatán (Zaldívar-Jiménez et al., 2010) y sistemas riparios en Jalisco (Allen, Santana-Michel, Arrona y Zedler, 2010). Cuatro trabajos abordaron la restauración de los bosques: 2 sobre bosque estacional (Williams-Linera y Álvarez-Aquino, 2010; Bonfil y Trejo, 2010), uno de bosque nublado (RamírezMarcial, González-Espinosa, Camacho-Cruz y Ortiz-Aguilar, 2010) y uno sobre el bosque de pino-encino (Rodríguez-Trejo y Myers, 2010).

Con la búsqueda realizada en WoS y SCOPUS hasta diciembre de 2016 se registraron 79 y 110 artículos, respectivamente, con autores de adscripción en México. Eliminando las referencias repetidas y expandiendo y depurando la base se llegó a un total de 206 referencias, de las cuales el $78 \%$ correspondieron a artículos de investigación, el $18 \%$ a artículos de opinión y propuestas metodológicas, y el $4 \%$ fueron metaanálisis o revisiones. Las revistas en donde más se publicó la investigación realizada en México hasta diciembre de 2016 en orden descendente por número de artículos publicados fueron Restoration Ecology, Ecological Engineering, Botanical Sciences, Forest Ecology and Management, Ecological Restoration, Madera y Bosques y Plos One (fig. 3). Los artículos fueron publicados en coautorías con investigadores de Estados Unidos, España, Colombia, Reino Unido y Argentina, principalmente. En el 82\% de las publicaciones el primer autor tuvo su adscripción en una institución mexicana, y en el $81 \%$ de los casos estos autores fueron los corresponsales. En cuanto al género de los autores con adscripción en México, el 57\% correspondió a hombres y el $43 \%$ a mujeres.

Las 206 publicaciones consideradas fueron generadas en aproximadamente 50 instituciones, principalmente de educación superior y en los centros de investigación del sistema Conacyt. Las principales afiliaciones de los autores, en orden descendente por número de artículos publicados, fueron 5 instituciones de la Universidad Nacional Autónoma de México (UNAM): Centro de Investigaciones en Ecosistemas (CIECO; ahora Instituto de Investigaciones en Ecosistemas y Sustentabilidad, IIES), Instituto de Ecología, Facultad de Ciencias e Instituto de Biología. Le siguen en importancia el Instituto de Ecología, A.C., la Universidad Autónoma del Estado de Morelos, El Colegio de la Frontera Sur, la Universidad Veracruzana, el Centro de Investigaciones Biológicas del Noroeste, la Universidad Autónoma de Nuevo León, la Universidad Michoacana de San Nicolás de Hidalgo y el Colegio de Postgraduados. En cuanto a la proporción de 


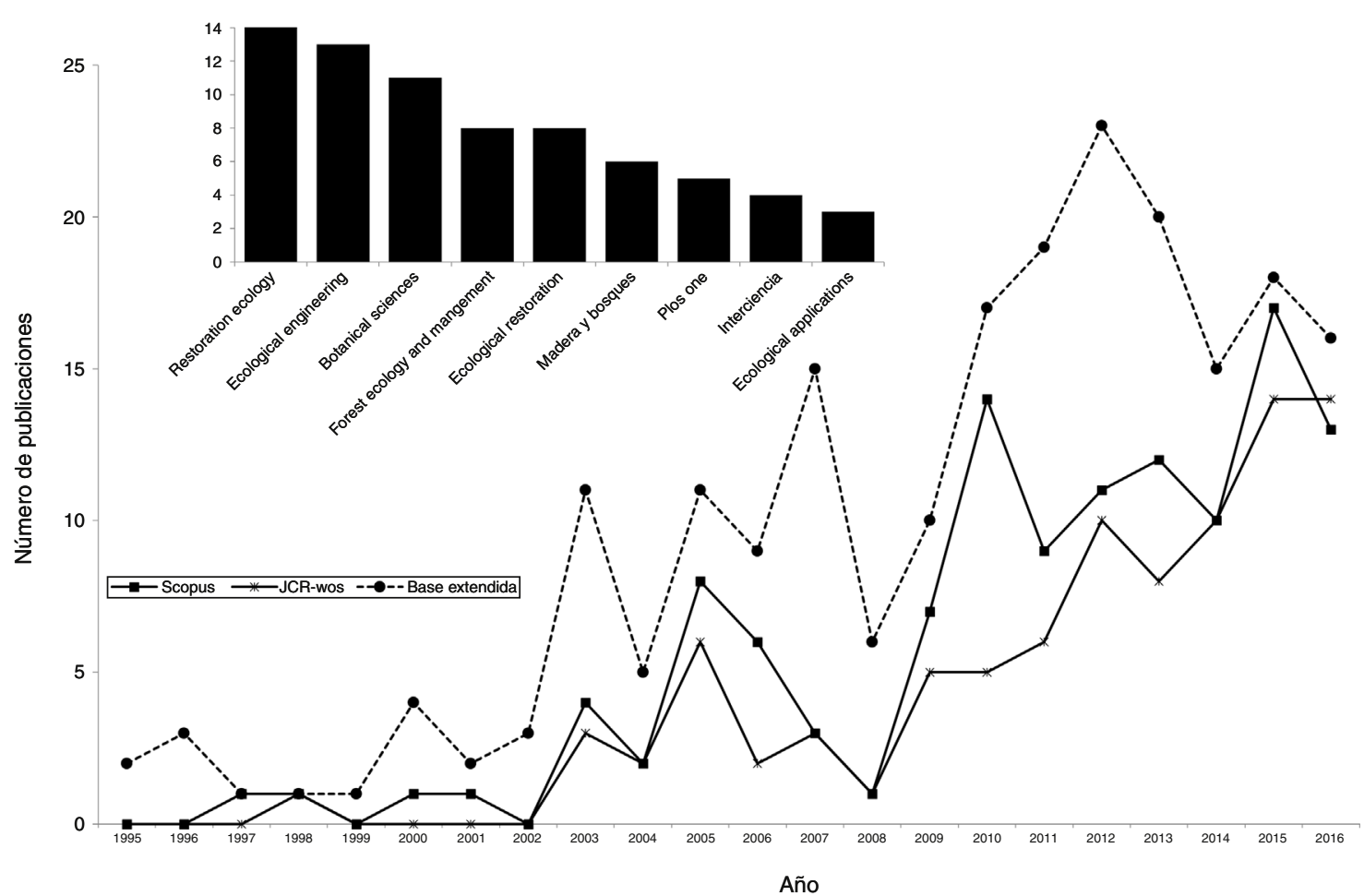

Figura 3. Crecimiento en el número de publicaciones según la base de datos SCOPUS (consulta: título (restor* or rehab*) Y título+resumen+palabras clave (ecolo*)), la base JCR-WoS (consulta: títutlo (restor* or rehab*) y tema (ecolo*)) y la base extendida considerando artículos encontrados en el google académico y REDALYC (1995-2016). Se muestran los artículos en los que algún autor tuvo su adscripción en una institución mexicana. El gráfico de barras muestra el número de artículos por revistas donde más se publican los artículos según el SCOPUS y JCR-WoS.

investigadores que en su página de internet mencionan en su línea de investigación a la ecología de la restauración o la restauración de ecosistemas, encontramos que en el IIES son el $23 \%$ de 31 investigadores, en el IE-UNAM son el $17 \%$ de 41 , en el INECOL son el $11 \%$ de 114 y en ECOSUR son el $9 \%$ de 149. Los porcentajes de investigadores dedicados a la restauración fueron estimaciones, considerando que tanto el número de investigadores como las líneas de investigación de las páginas de los investigadores dependen de la actualización de cifras y datos que hacen las instituciones en sus portales.

\section{Los principales ecosistemas estudiados}

El mayor número de artículos publicados se refirieron a la restauración de especies leñosas de las selvas húmedas, bajas y medianas caducifolias, bosques de coníferas y encinares y bosques mesófilos de montaña, con 30, 28, 23 y 13 artículos, respectivamente (fig. 4). En conjunto, el $71 \%$ de los estudios fueron sobre los ecosistemas forestales (sin considerar los manglares). Es interesante notar que existen en las investigaciones la incorporación de 2 o más grupos biológicos o estudios multitaxonómicos (p. ej., Allen, Allen y Gómez-Pompa, 2005; De la Peña-Domene, Martínez-Garza, Palmas-Pérez, Rivas-Alonso y Howe, 2014; Hernández, Boege, Lindig-Cisneros y del Val, 2014; Huante et al., 2012; López-Rosas et al., 2010; San José, Garmendia y Cano-Santana, 2013), aunque fue siempre en menor proporción que los estudios enfocados en la recuperación de árboles y arbustos (fig. 4).

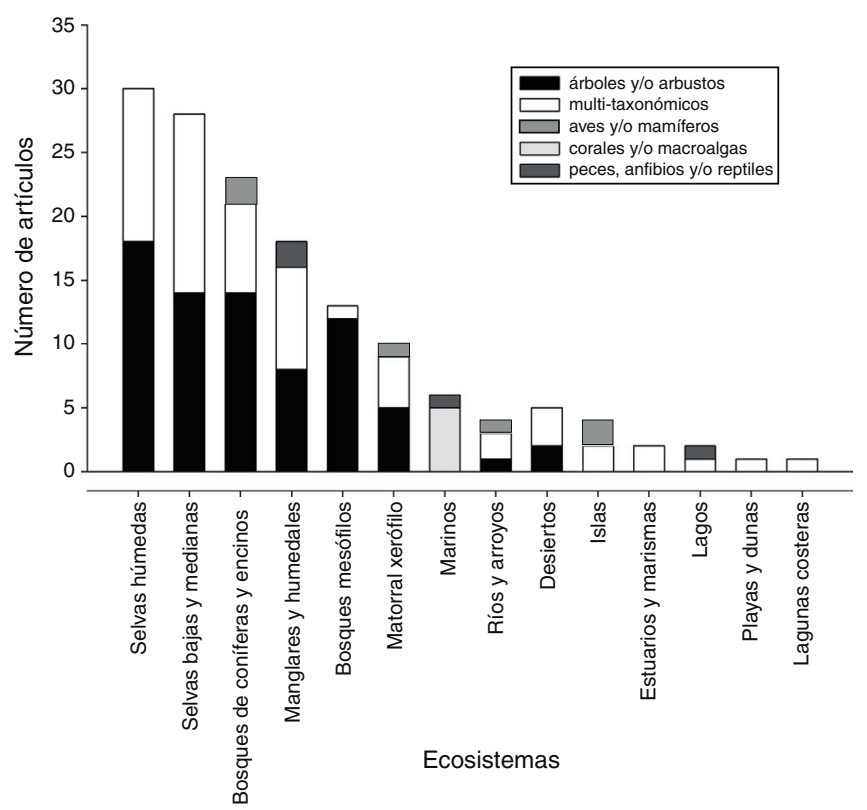

Figura 4. Principales ecosistemas y grupos biológicos evaluados en investigaciones sobre ecología de la restauración en México. Se seleccionaron 147 investigaciones de los 206 artículos publicados, los cuales abordaron explícitamente la restauración de algún ecosistema.

Se registraron 18 estudios sobre manglares y humedales, mientras que pocas investigaciones (6 estudios) fueron sobre la restauración de ecosistemas marinos (fig. 4). Los resultados mostraron que existen esfuerzos notables con 
respecto a la recuperación de la topografía, el hidroperíodo, la geomorfología, la flora y la fauna en los humedales herbáceos (Angeler, Chow-Fraser, Hanson, Sánchez-Carrillo y Zimmer, 2003; Flores-Verdugo et al., 2007; Lindig-Cisneros, Desmond, Boyer y Zedler, 2003; López-Rosas et al., 2010; Zepeda-Gómez, Lot, Antonio-Nemiga y Manjarrez, 2015), manglares (Bashan, Moreno, Salazar y Álvarez, 2013; BenítezPardo et al., 2015; Holguin, Vázquez y Bashan, 2001; Olguín, Hernández y Sánchez-Galván, 2007; Reyes-Chargoy y TovillaHernández, 2002; Toledo, Rojas y Bashan, 2001; Vovides, Bashan, López-Portillo y Guevara, 2011; Zaldívar-Jiménez et al., 2010), dunas (Lithgow et al., 2013; Moreno-Casasola, Martínez y Castillo-Campos, 2008) y lagunas costeras (Hansen, van Afferden y Torres-Beiarano, 2007; Yáñez-Arancibia et al., 2014). La restauración de los servicios ambientales de los humedales se ha abordado en forma de metaanálisis (Meli, Rey-Benayas, Balvanera y Martínez-Ramos, 2014). La restauración de las zonas áridas, semiáridas y desiertos también ha sido poco abordada (fig. 4) y se ha enfocado principalmente en estudiar técnicas para aminorar los factores limitantes para el establecimiento de las plantas y en la introducción de especies nodrizas (Antonio-Garcés, Peña, Cano-Santana, Villeda y Orozco-Segovia, 2009; Bacilio, Hernández y Bashan, 2006; Encino-Ruiz, Lindig-Cisneros, Gómez-Romero y BlancoGarcía, 2013; Foroughbakhch et al., 2006; López-Lozano, Carcaño-Montiel y Bashan, 2016; Mendoza-Hernández y CanoSantana, 2009; Ureta y Martorell, 2009).

La ecología de la restauración de lagos estuvo mínimamente representada (Ramírez-Herrejón et al., 2014), así como la restauración de macroalgas (Hernández-Carmona, García, Robledo y Foster, 2000), arrecifes (Islas-Peña, Liñán-Cabello y Torres-Orozco, 2004, Liñan-Cabello et al., 2011; TortoleroLangarica, Cupul-Magaña y Rodríguez-Troncoso, 2014) y ríos (Mazari-Hiriart et al., 2014; Ramírez-Hernández, HinojosaHuerta, Peregrina-Llanes, Calvo-Fonseca y Carrera-Villa, 2013; Salinas-Rodríguez y Ramírez-Marcial, 2010). Cabe destacar los proyectos de restauración en islas que se han enfocado en la remoción de especies exóticas o de comportamiento invasivo para favorecer la recuperación de la biota nativa, que han sido ejemplo de restauración a nivel mundial dados sus resultados y la escala de sus acciones (Aguirre-Muñoz et al., 2011).

Los enfoques menos abordados han sido los aspectos genéticos y demográficos de la restauración (Lara-Cabrera, Alejandre-Melena, Medina-Sánchez y Lindig-Cisneros, 2009; Arias-Medellín, Bonfil y Valverde, 2016), interacciones ecológicas (Juan-Baeza, Martínez-Garza y del Val, 2015; Villa-Galaviz, Boege y del Val, 2012), el estudio de la fauna como catalizador e indicador de la restauración (De la Peña-Domene et al., 2014; González-Tokman y Martínez-Garza, 2015; Hernández-Flores, Osorio-Beristain y Martínez-Garza, 2016; Hernández-Ladrón de Guevara, Rojas-Soto, López-Barrera, Puebla-Olivares y Díaz-Castelazo, 2012; MacGregor-Fors, Blanco-García y Lindig-Cisneros, 2010; San José et al., 2013), así como la importancia de la diversidad filogenética en la selección de los ensambles (Campbell, Freeman, Emlen y López-Ortiz, 2010; Verdú, Gómez-Aparicio y Valiente-Banuet, 2012).

\section{Ecología de la restauración en simposios y congresos}

En noviembre de 2014 se llevó a cabo el Primer Simposio Nacional de Restauración Ecológica, que tuvo como sede la Universidad Autónoma del Estado de Morelos. Este simposio fue planeado como parte de las actividades de la Estrategia Mexicana de Conservación Vegetal (EMCV), coordinada en México por la Conabio; en particular, lo referente al objetivo 3 , que llama a la rehabilitación o restauración, para 2030, al menos del $50 \%$ de las áreas terrestres y marítimas degradas (Conabio, 2012). Las actividades de la EMCV dieron inicio con un taller donde académicos, instituciones gubernamentales y restauradores se dieron cita para planear las acciones de la Estrategia Global de Conservación Vegetal (Conabio, 2012). Tomando como base las recientes publicaciones científicas, el Primer Simposio Mexicano de Restauración Ecológica se llevó a cabo con 25 ponentes de 17 organizaciones académicas gubernamentales y organizaciones civiles. Los participantes, con experiencia en aspectos sociales, experimentales y prácticos de la restauración ecológica, abordan la restauración en diferentes ecosistemas de México: bosque nublado (4 trabajos), bosque templado (3), matorrales (2), selva húmeda (3), selva estacional (4), dunas costeras (2), mientras que de manglar, islas, humedal urbano, arrecifes y agua y multidisciplina se presentó uno de cada tema (Ceccon, Barrera-Cataño, Aronson y Martínez-Garza, 2015). El tema de la mayoría de las presentaciones invitadas fue acerca de estudios biológicos experimentales (52\%), mientras que solo una quinta parte se relacionaba con los componentes sociales, por ejemplo, la participación de los dueños de la tierra; finalmente, un pequeño porcentaje de estudios incluía aspectos prácticos (17\%). En este evento se registraron 316 asistentes y se presentaron 74 carteles, además de 2 conferencias magistrales. Derivado de este simposio se publicó el libro titulado «Experiencias mexicanas en la restauración de ecosistemas» (Ceccon y Martínez-Garza, 2016).

A partir de 2006 comenzaron los congresos de la recién creada Sociedad Científica Mexicana de Ecología. En el congreso realizado en 2008 en la ciudad de Mérida, Yucatán, se presentó el más alto porcentaje de trabajos sobre restauración con respecto a todos los trabajos presentados en el congreso, mientras que en 2011, en Boca del Río, Veracruz, aunque solo se organizó un simposio, se registró el mayor número de ponencias orales y carteles sobre este tema ( 31 y 26 , respectivamente; fig. 5).

\section{Formación de recursos humanos}

En cuanto a la formación de recursos humanos, en todo el país se cuenta solamente con una licenciatura en Ingeniería en Restauración Forestal, ofrecida por la Universidad Autónoma de Chapingo; un diplomado técnico en Restauración Ambiental, que se ofrece como opción a partir del quinto semestre de la licenciatura en Ciencias Ambientales impartida por la Escuela Nacional de Estudios Superiores Morelia (ENESMorelia, UNAM), y una maestría en Restauración Ecológica, enfocada en ecosistemas costeros, ofrecida por la Universidad Autónoma del Carmen. El Instituto de Ecología A.C. 


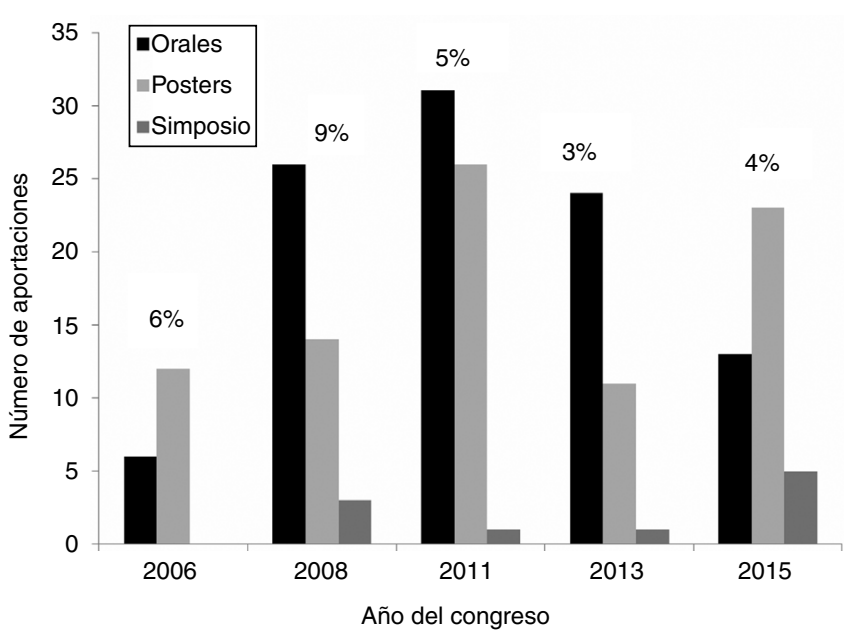

Figura 5. Presentaciones de posters, conferencias y número de simposios organizados de ecología de la restauración en los congresos de ecología de 2006 a 2014. El porcentaje mostrado indica el número de posters y presentaciones orales con respecto al total de aportaciones registradas en el congreso en el 2006 (287 aportaciones), 2008 (463), $2011(1,200), 2013(1,072)$ y 2015 (996).

(INECOL), El Colegio de la Frontera Sur (ECOSUR), FESZaragoza, UNAM y el Instituto Potosino de Investigación Científica y Tecnológica (IPICYT) ofrecen y avalan académicamente diplomados especializados de restauración, algunos en conjunto con organizaciones no gubernamentales, como Pronatura y La Fundación Internacional para la Restauración de Ecosistemas (FIRE).

En la UNAM ha habido importantes esfuerzos para la formación de recursos humanos especializados en restauración ecológica. En 2001 se publicó la primera convocatoria de la maestría en Ciencias Biológicas con orientación en Restauración Ecológica. En esta maestría se formaron alrededor de 100 alumnos durante los 10 años que estuvo activa y el $24 \%$ de ellos han publicado sus resultados en revistas indexadas. En 2012 egresó la última generación de esta maestría y más tarde el programa quedó incluido como un campo de conocimiento del nuevo posgrado en Ciencias de la Sostenibilidad (PCS) de la UNAM (http://www.posgrado.unam.mx/sostenibilidad/index.html; consultado en junio de 2015). El PCS busca formar profesionales que contribuyan al desarrollo sostenible del país. La mayor innovación de este posgrado es que integra las ciencias naturales, las sociales, la ingeniería y el urbanismo. El PCS fue aprobado por el Consejo Universitario en su sesión del 24 de marzo de 2015, y el mismo año se abrió la primera convocatoria para el ingreso de los alumnos. Las 3 materias obligatorias que antes se ofrecían en la maestría de Restauración ahora forman parte del nuevo plan de estudios del posgrado en Sustentabilidad para el campo de conocimiento sobre Restauración Ambiental. El PCS tiene como objetivo fundamental formar expertos con una perspectiva transdisciplinaria, lo que beneficiará al campo de la restauración ambiental. Los esfuerzos iniciados para la formación de una masa crítica que hiciera frente a los problemas ambientales en México en 2001 con la maestría en Ciencias Biológicas con orientación en Restauración Ecológica están ahora fortaleciendo al PCS.

\section{Financiamiento}

Con la revisión de los proyectos de investigación financiados por el programa de Ciencia Básica del Conacyt se registraron en total 64 proyectos (fig. 6), de los cuales 45 se relacionaron a la biotecnología y remediación, 2 con aspectos sociales de la restauración (ética ambiental y organización social) y 17 fueron de ecología de la restauración de ecosistemas forestales y costeros (13 y 4 proyectos, respectivamente). Los proyectos financiados cada año representaron entre el 0.5 y el $2 \%$ del total de proyectos apoyados en cada convocatoria. En el caso de los proyectos financiados por el Programa de Apoyo a Proyectos de Investigación e Innovación Tecnológica (PAPIIT) de la UNAM entre 2012 y 2015, las propuestas relacionadas con la ecología de la restauración representan el $0.46 \%$ del total apoyadas en dicho período. Se revisaron 11 proyectos trianuales financiados, y el $55 \%$ estuvieron enfocados a ecología de la restauración de ecosistemas forestales, abordando diferentes aspectos como el de cambio climático, interacciones ecológicas y migración asistida. Solo un proyecto abordó la restauración de ecosistemas costeros (manglares), y el resto (36\%) tuvieron temáticas sociales (organización comunitaria e integración de la población local en la restauración). En cuanto a los proyectos apoyados por Conabio, el $21 \%$ de los proyectos del programa de compensación y restauración ambiental fueron ejecutados por instituciones académicas (Arriaga, 2014). De estos 26 proyectos, 8 fueron explícitamente de ecología de la restauración y la mayoría fueron sobre ecosistemas lagunares, de manglar y humedales; esto respondió a convocatorias especiales para restaurar este tipo de ecosistemas (anexo 1 en Arriaga, 2014). Si consideramos todos los programas de financiamiento de Conabio, se encontraron 55 proyectos que tuvieron una temática de restauración; el número de proyectos no mostró un incremento temporal, ya que respondieron a convocatorias especiales enfocadas principalmente a financiar la restauración de ecosistemas acuáticos como manglares, arrecifes, humedales, playas, islas y lagunas costeras (fig. 7).

\section{Posición relativa de México en un contexto latinoamericano}

En México, de 1999 a 2014 se registró un incremento del $600 \%$ en la tasa anual de publicaciones indexadas sobre el tema de restauración (WoS y SCOPUS). Con respecto a Latinoamérica, México ocupa el segundo lugar después de Brasil (fig. 8), que en ese mismo periodo registró un incremento del 1,500\% en su tasa anual de publicaciones. El número acumulado de publicaciones (SCOPUS) para Brasil fue de 207, mientras que para México fue de 110; Costa Rica, Colombia, Argentina y Chile siguieron con 30, 30, 29 y 29 trabajos, respectivamente.

El impacto de la ecología de la restauración en México también se ha visto reflejado en la producción de libros y manuales. Como referencia, de los 182 títulos en inglés, español y portugués publicados hasta el año 2014, el $15 \%$ tuvo autores o editores con afiliación en México; estos representaron el $42 \%$ de la producción total en Latinoamérica. Un total de 18 libros y 11 guías se registraron hasta finales de 2016; dentro de las guías dominaron los manuales que generan la información base y estrategias 


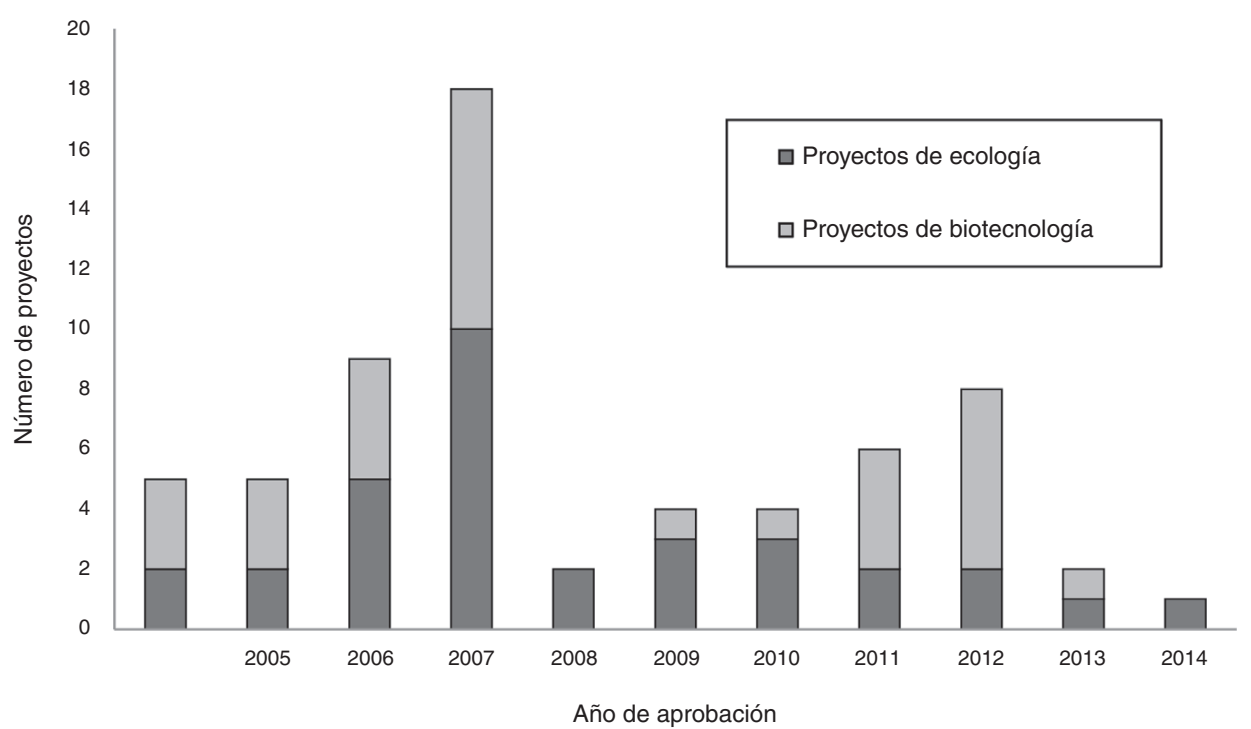

Figura 6. Proyectos aprobados de la Convocatoria de Investigación Ciencia Básica (Conacyt) 2004-2014 relacionados con la ecología de la restauración y aquellos relacionados con la biotecnología o remediación de suelo y agua.

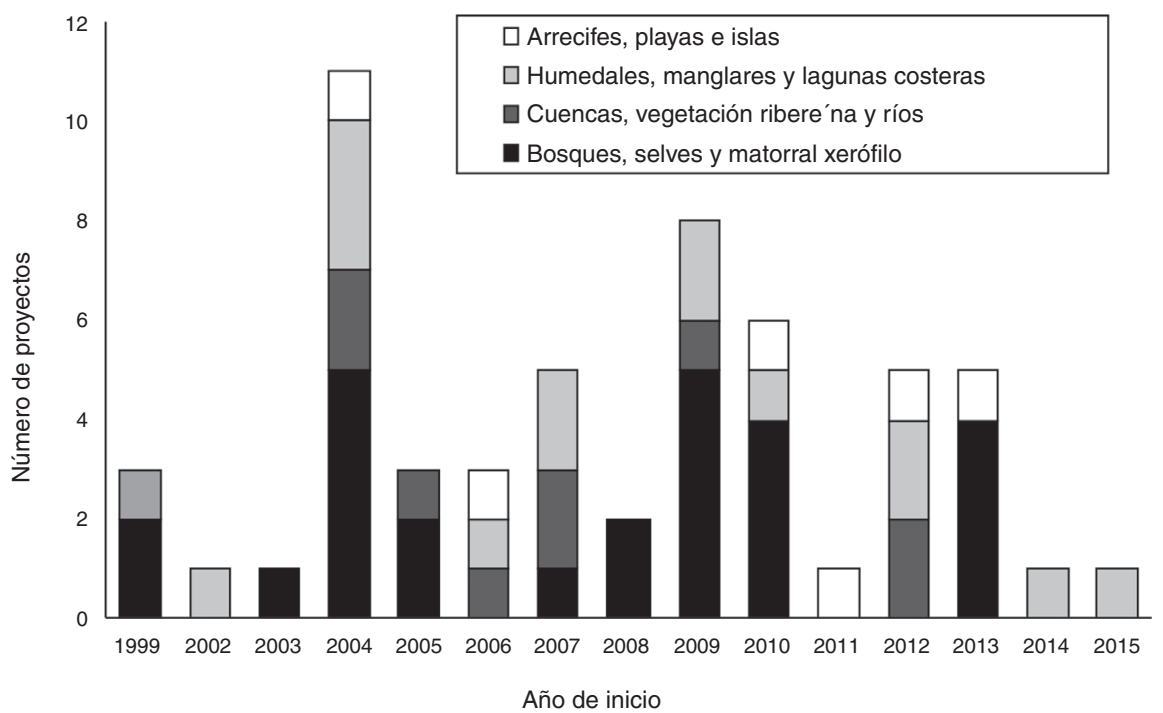

Figura 7. Proyectos de restauración financiados por Conabio. Se realizó una depuración seleccionando finalmente 55 proyectos de restauración y ecología de la restauración ejecutados por diferentes tipos de actores (académico, ONG, gobierno, individuos, empresas).

Fuente: http://www.conabio.gob.mx/web/proyectos/proyectos_financiados.html [consultado Jun 2015].

para la restauración de ecosistemas forestales (Arriaga, Cervantes y Vargas-Mena, 1994; Benítez-Badillo, Pulido-Salas y Equihua-Zamora, 2004; Douterlungne y Ferguson, 2012; Meli y Carrasco-Carballido, 2011; Niembro, Sánchez y Vásquez, 2010; Ramírez-Marcial et al., 2012), y en menor medida estuvieron los que muestran técnicas de rehabilitación y monitoreo de sitios afectados por la minería (Fragoso y Rojas-Fernández, 2012; Jiménez, Huante y Rincón, 2006), de reforestación de médanos (Moreno-Casasola, Infante, Travieso-Bello y MaderoVega, 2009), de manglares (Agraz-Hernández et al., 2007) y de ríos urbanos (González-Reynoso, Hernández Muñoz, Perló y Zamora, 2010). En cuanto a los libros, destacan aquellos sobre los fundamentos ecológicos (Oyama y Castillo, 2006; Sánchez et al., 2005), los fundamentos sociales (Ceccon y Pérez, 2016) la restauración de dunas (Martínez, Gallego-Fernández y Hesp,
2013), de bosques (Ceccon, 2013; González-Espinosa, ReyBenayas y Ramírez-Marcial, 2008) y de islas (Santos-del Prado y Peters, 2008).

\section{Discusión}

Actualmente, dada la crisis ambiental, se han establecido convenios internacionales con metas muy ambiciosas. Particularmente México, como signatario del Convenio de la Diversidad Biológica (CDB), está comprometido a restaurar para 2020 todos los ecosistemas prioritarios (meta AICHI 14) y el $15 \%$ de los ecosistemas degradados (meta AICHI 15; Conabio, 2014). Por otro lado, la Estrategia Mexicana para la Conservación de la Diversidad Vegetal (Conabio, 2012) establece restaurar el 50\% de los ecosistemas degradados para 2030. Recientemente, en 


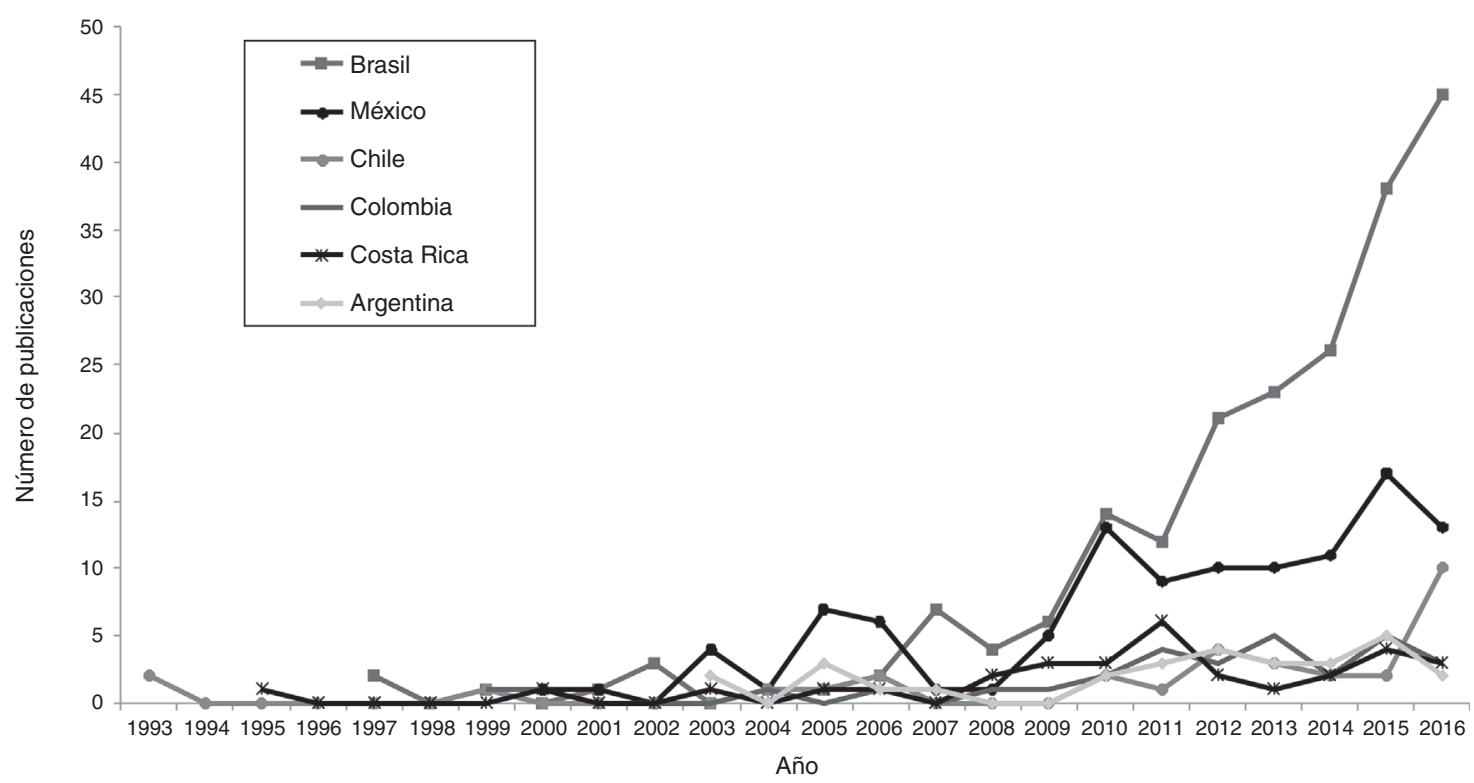

Figura 8. Crecimiento en el número de publicaciones según la base de datos SCOPUS (consulta: título [(restor* or rehab*) Y título+resumen+palabras clave (ecolo*)] y seleccionando países de Latinoamérica (1993-2016).

Lima, Perú (COP20-2014), México se comprometió a iniciar la restauración en 8.5 millones de ha de su territorio para el año 2020 como parte de la iniciativa $20 \times 20$ para Latinoamérica (IUCN, 2014). Aun con estos grandes compromisos, nuestro país no cuenta con una legislación sólida sobre el tema (Ceccon et al., 2015a; Carabias, Ruíz y Rabasa, 2016), ni con un plan nacional, ni con un fondo nacional de restauración para los proyectos de largo plazo y de gran escala. En este contexto de pocos recursos financieros y grandes compromisos, las instituciones públicas han comenzado a interactuar con centros de investigación para la generación de mapas que indiquen a nivel nacional dónde es más urgente y factible restaurar e implementar políticas públicas para hacer más eficiente la restauración (Tobón, Koleff, Urquiza-Haas y GarcíaMéndez, 2016). Bajo este contexto, la restauración de paisajes prevé la necesidad de utilizar análisis jerárquicos para priorizar especies (Gelviz-Gelvez, Pavón, Illoldi-Rangel y BallesterosBarrera, 2015; Meli, Martínez-Ramos y Rey-Benayas, 2013, Meli, Martínez-Ramos, Rey-Benayas y Carabias, 2014), estrategias (Birch et al., 2010; Latofski-Robles, Aguirre-Muñoz, Méndez-Sánchez, Reyes-Hernández y Schlüter, 2014; Trabucchi, Puente, Comin, Olague y Smith, 2012; Ceccon, González y Martorell, 2015) y áreas en las que sea no solo necesario, sino también factible, restaurar (Adame, Hermoso, Perhans, Lovelock y Herrera-Silveira, 2015; Cirelli y Sánchez-Cordero, 2009; González-Ovando, Plascencia-Escalante y Martínez-Trinidad, 2016; Lithgow, Martínez y Gallego-Fernández, 2015; Newton et al., 2012; Uribe, Geneletti, del Castillo y Orsi, 2014).

La restauración ecológica en México tiene una dimensión social que determina la factibilidad de cualquier esfuerzo desde la ecología de la restauración (Ceccon y Pérez, 2016). La mayor parte del territorio nacional (cerca del 90\%) es propiedad privada y social (51\% son ejidos; Sedatu, 2013), y gran parte de los recursos asignados a la ecología de la restauración provienen de fondos públicos (ver Ceccon et al., 2015a). La existencia de un México rural formado por pequeñas localidades que ocupan más de la mitad del territorio y concentran una gran riqueza natural, y por otra parte un México urbano con una alta densidad de población que requiere mayor acceso a servicios e infraestructura, impone grandes retos a la restauración ecológica. En este escenario la restauración se considera como «una construcción social en donde deben legitimarse tanto las necesidades de recuperar la estructura y función de los ecosistemas, como las necesidades y aspiraciones de desarrollo óptimo de los diferentes grupos sociales involucrados» (Ramírez-Marcial, GonzálezEspinosa, Musálem-Castillejos, Savelli y Gómez-Pineda, 2014); lo anterior involucra no solo la recuperación del capital natural (Aronson, Milton y Blignaut, 2007) sino también la del capital social (Bullock, Aronson, Newton, Pywell y Rey-Benayas, 2011).

Muchos estudios de ecología en México sirven de base o tienen implicaciones para la ecología de la restauración; sin embargo, la revisión de literatura realizada en este estudio fue estricta en sus criterios de búsqueda con el fin de no sobreestimar las tendencias sobre el desarrollo del tema en su componente científico y su aporte al fortalecimiento de la ecología de la restauración a nivel global. El establecimiento de bases de datos sobre capítulos de libro y tesis de pregrado y posgrado en México permitiría complementar los resultados mostrados en este estudio. Para el avance de la ecología de la restauración es prioritario establecer bases científicas de libre acceso, como una base de datos de experiencias exitosas y no exitosas de prácticas de restauración para cada ecosistema.

En el presente estudio se muestra una tendencia creciente de publicaciones de ecología de restauración en nuestro país. También se ha revelado una autonomía en la generación del conocimiento, ya que los grupos de trabajo con afiliación en México se encuentran liderando la mayor parte de la investigación que se realiza en nuestro país. Esta tendencia creciente responde también a un incremento de publicaciones 
científicas por parte de los científicos mexicanos que en la última década incrementaron en un $65 \%$ la producción de artículos JCR-WoS, principalmente en el área de agricultura, ciencias de la tierra y biología (Gaze y Breen, 2014). Sin embargo, al comparar la producción de artículos de ecología de la restauración con respecto a Brasil, es evidente que el crecimiento en la producción de artículos no ha sido tan acelerado ni suficiente para responder a la necesidad de revertir en lo posible el daño ambiental. En cuanto a la representación por género, la proporción de mujeres investigadoras trabajando en la ecología de la restauración fue casi el doble (43\%) comparada con la proporción de mujeres que han publicado en el área de ecología y evolución en el mundo entre 1542 y 2011 (23\%; West, Jacquet, King, Correll y Bergstrom, 2013).

En México hace falta un plan nacional de restauración, como el que ya existe en Colombia (Murcia y Guariguata, 2014) y en Ecuador (MAE, 2014), y una política científica que acompañe dicho plan. Los primeros pasos para desarrollar este plan nacional de restauración ya se han dado con la realización del taller «Retos y perspectivas para cumplir los acuerdos internacionales en materia de Restauración Ecológica» que se realizó en noviembre de 2015, apoyado por Conabio y el Centro de Investigación Forestal Internacional (CIFOR, por sus siglas en inglés, www.cifor.org). El objetivo principal del taller fue planear la generación de los principales insumos para el plan nacional de restauración: los mapas de sitios prioritarios para restauración y el protocolo para hacer una evaluación nacional de los proyectos de restauración de los últimos 20 años trabajando con las instituciones gubernamentales, la academia, las organizaciones de la sociedad civil y las fundaciones privadas. Por otra parte, México no cuenta con un esquema de financiamiento a largo plazo que estimule proyectos de gran escala. El número de proyectos relacionados con la restauración que han sido apoyados por fondos de investigación y por otros fondos, como los de Conabio, fue relativamente bajo considerando que entre el 9 y el $23 \%$ de los investigadores de una muestra de centros de investigación están incorporando este tema en sus líneas de investigación. El país tampoco cuenta con sistemas de evaluación que promuevan que los investigadores se comprometan a realizar investigación de largo plazo y que evalúen procesos ecológicos que requieren censos multianuales como muchos de los indicadores del éxito de la restauración. En cuanto a la formación de recursos humanos, es necesario incrementar los esfuerzos, ya que la oferta es muy escasa. El reto es que más instituciones en el país prioricen el tema en sus marcos normativos y programas de posgrado, incluyendo la multidisciplina en la formación de recursos humanos.

Aun cuando la Conafor ha reportado recientemente una meta de incrementar en 170,000 ha/año la superficie dedicada a la restauración de bosques entre 2013 y 2018 (Conafor, 2014) y figuramos en la lista internacional de los 10 países que más árboles siembran (FAO, 2010), la misma Conafor ha reconocido que esta cifra puede estar sobreestimada, ya que la información sobre la expansión de área de bosque es una aproximación obtenida de los registros anuales y las hectáreas apoyadas del Programa Nacional de Reforestación (apoyos para la conservación y restauración de ecosistemas forestales). Por otra parte, la Conafor reconoce no contar con evaluaciones a mediano y largo plazo del éxito de las plantaciones en el contexto de la restauración (FAO, 2015b). Esto muestra la falta de vinculación entre la teoría y la práctica extensiva. Dado que es urgente establecer un mapeo de actores relevantes en el proceso de restauración ecológica, en el taller antes mencionado se generó una lista de 81 actores relevantes para incorporarlos en el Plan Nacional de Restauración con el fin de coordinar esfuerzos y generar conocimiento ecológico.

Para acompañar estos procesos es imperativo promover el desarrollo de la ecología de la restauración en nuestro país, el cual requiere un enfoque multidisciplinario para tener un impacto mayor en la práctica, ya que los contextos socioeconómicos particulares en cada sitio imponen la necesidad de diseñar metas de restauración junto con la población local para asegurar la generación de servicios ambientales y la persistencia a largo plazo de las prácticas para la recuperación de ecosistemas.

\section{Agradecimientos}

El presente trabajo es un producto del simposio «La ecología en México: retos y perspectivas», organizado en 2014 por la Sociedad Científica Mexicana de Ecología. Agradecemos a las personas que apoyaron la edición de este documento: Sandra Daniela Hernández, María de los Ángeles García, Constanza Pinto Merchant, Jorge Córdova Nieto y Lizbeth González. Gracias a Jorge López-Portillo, Patricia Moreno-Casasola y Javier Laborde por la información ofrecida sobre la ecología de la restauración en ecosistemas particulares y a Georgina García Méndez por la información sobre la maestría con orientación en Restauración Ecológica. F. López-Barrera agradece el apoyo para estancia sabática en el extranjero, convocatoria 2016, Conacyt (121652), E. Ceccon agradece al proyecto PAPIIT IN300615.

\section{Referencias}

Adame, M. F., Hermoso, V., Perhans, K., Lovelock, C. E. y Herrera-Silveira, J. A. (2015). Selecting cost-effective areas for restoration of ecosystem services. Conservation Biology, 29, 493-502.

Agraz-Hernández, C., Osti-Sáenz, J., Jiménez-Zacarías, J., García-Zaragoza, C., Arana-Lezama, R., Chan-Canul, E., et al. (2007). Guía técnica: criterios para la restauración del mangle. Campeche, México: Universidad Autónoma de Campeche, Comisión Federal de Electricidad, Comisión Nacional Forestal.

Aguirre-Muñoz, A., Mendoza-Alfaro, R., Arredondo-Ponce, B. H., Arriaga Cabrera, L., Campos-González, E., Contreras-Balderas, S., et al. (2009). Especies exóticas invasoras: impactos sobre las poblaciones de flora y fauna, los procesos ecológicos y la economía. En J. Sarukhán (Ed.), Capital natural de México. Vol. II: Estado de conservación y tendencias de cambio (pp. 277-318). Ciudad de México: Conabio.

Aguirre-Muñoz, A., Samaniego-Herrera, A., Luna-Mendoza, L., Ortiz-Alcaraz, A., Rodríguez-Malagón, M., Méndez-Sánchez, F., et al. (2011). Island restoration in Mexico: ecological outcomes after systematic eradications of invasive mammals. En C. R. Veitch, M. N. Clout, y D. R. Towns (Eds.), Island invasives: eradication and management (pp. 250-258). Gland, Switzerland: IUCN.

Allen, M. F., Allen, E. B. y Gómez-Pompa, A. (2005). Effects of mycorrhizae and non-target organisms on restoration of a seasonal tropical forest in Quintana Roo, Mexico: Factors limiting tree establishment. Restoration Ecology, 13, $325-333$. 
Allen, A. E., Santana-Michel, F. J., Arrona, C. O. y Zedler, J. B. (2010). Integrating ecological and ethnobotanical priorities into riparian restoration. Ecological Restoration, 28, 377-388.

Álvarez-Aquino, C. y Williams-Linera, G. (2012). Seedling survival and growth of tree species: Site condition and seasonality in tropical dry forest restoration. Botanical Sciences, 90, 341-351.

Álvarez-Aquino, C., Williams-Linera, G. y Newton, A. C. (2004). Experimental native tree seedling establishment for the restoration of a Mexican cloud forest. Restoration Ecology, 12, 412-418.

Álvarez-Sánchez, F. J., Guadarrama-Chávez, M. P., Sánchez-Gallén, I. y Olivera, D. (2007). Restauración de ambientes deteriorados derivados de la selva húmeda: el uso de los hongos micorrizógenos arbusculares. Boletín de la Sociedad Botánica de México, 80, 59-68.

Álvarez-Sánchez, J., Sánchez-Gallén, I. y Guadarrama, P. (2009). Analyses of ecophysiological traits of tropical rain forest seedlings under arbuscular mycorrhization: implications in ecological restoration. En A. Varma y A. C. Kharkwal (Eds.), Symbiotic Fungi: principles and practice (pp. 293-305). Berlin: Springer-Verlag.

Angeler, D. G., Chow-Fraser, P., Hanson, M. A., Sánchez-Carrillo, S. y Zimmer, K. D. (2003). Biomanipulation: a useful tool for freshwater wetland mitigation? Freshwater Biology, 48, 2203-2213.

Antonio-Garcés, J., Peña, M., Cano-Santana, Z., Villeda, M. y Orozco-Segovia, A. (2009). Cambios en la estructura de la vegetación derivados de acciones de restauración ecológica en las Zonas de Amortiguamiento Biológicas y Vivero Alto. En A. Lot y Z. Cano-Santana (Eds.), Biodiversidad del ecosistema del pedregal de San Ángel (pp. 465-481). México D.F.: UNAM

Arias-Medellín, L. A., Bonfil, C. y Valverde, T. (2016). Demographic analysis of Agave angustifolia (Agavaceae) with an emphasis on ecological restoration. Botanical Sciences, 94, 513-530.

Aronson, J., Milton, S. J., y Blignaut, J. (Eds.). (2007). Restoring Natural Capital: science, business and practice. Washington, D.C.: Island Press.

Arriaga, M. V. (2014). Evaluación del impacto y logros alcanzados en restauración y conservación ambiental de los proyectos financiados a través del Programa de Restauración y Compensación Ambiental (1.- etapa). México D.F.: Centro Interdisciplinario de Biodiversidad y Ambiente A.C. Informe final SNIB-Conabio, proyecto No. LP001.

Arriaga, M. V., Cervantes, V. y Vargas-Mena, A. (1994). Manual de reforestación con especies nativas: colecta y preservación de semillas, propagación y manejo de plantas. México D.F.: Instituto Nacional de Ecología, Sedesol.

Avendaño-Yáñez, M., Sánchez-Velásquez, L. R., Meave, J. A. y Pineda-López, M. R. (2014). Is facilitation a promising strategy for cloud forest restoration? Forest Ecology and Management, 329, 328-333.

Bacilio, M., Hernández, J. P. y Bashan, Y. (2006). Restoration of giant cardon cacti in barren desert soil amended with common compost and inoculated with Azospirillum brasilense. Biology and Fertility of Soils, 43, 112-119.

Badano, E. I., Pérez, D. y Vergara, C. H. (2009). Love of nurse plants is not enough for restoring oak forests in a seasonally dry tropical environment. Restoration Ecology, 17, 571-576.

Badano, E. I., Samour-Nieva, R. O. y Flores, J. (2011). Emulating nurse plants to restore oak forests. Ecological Engineering, 37, 1244-1248.

Baena, M. L. y Halffter, G. (2008). Extinción de especies. En J. Sarukhán (Ed.), Capital natural de México. Vol. I. Conocimiento actual de la biodiversidad (pp. 263-282). México D.F.: Conabio.

Bashan, Y., Moreno, M., Salazar, B. G. y Álvarez, L. (2013). Restoration and recovery of hurricane-damaged mangroves using the knickpoint retreat effect and tides as dredging tools. Journal of Environmental Management, 116, 196-203.

Benítez-Badillo, G., Pulido-Salas, M. T. y Equihua-Zamora, M. (2004). Árboles multiusos nativos de Veracruz para reforestación, restauración y plantaciones. Xalapa: INECOL, A.C., SIGOLFO, Conafor.

Benítez-Pardo, D., Flores-Verdugo, F. J., Casas-Valdez, M., HernándezCarmona, G., Valdez-Hernández, J. I. y Gómez-Muñoz, V. (2015). Forestación de isletas de dragado utilizando dos especies de mangles, en una laguna costera del Golfo de California, México. Botanical Sciences, 93, $165-174$.

Birch, J. C., Newton, A. C., Álvarez Aquino, C., Cantarello, E., Echeverría, C., Kitzberger, T., et al. (2010). Cost-effectiveness of dryland forest restoration evaluated by spatial analysis of ecosystem services. Proceedings of the National Academy of Sciences of the United States of America, 107, 21925-21930.

Blanco-García, A., Sáenz-Romero, C., Martorell, C., Alvarado-Sosa, P. y LindigCisneros, R. (2011). Nurse-plant and mulching effects on three conifer species in a Mexican temperate forest. Ecological Engineering, 37, 994-998.

Bollo-Manent, M., Hernández-Santana, J. y Méndez-Linares, A. (2014). The state of the environment in Mexico. Open Geosciences, 6, 219-228.

Bonfil, C., Fernández y Fernández, D. y González-Espinosa, M. (2015). Un primer análisis del estado actual de los estudios de restauración ecológica en México. En B. Brow (Ed.), Tzintzuntzan, el lugar de los colibríes-otra vez (pp. 30-39). Morelia: Museo de Arte Contemporáneo Alfredo Zalce.

Bonfil, C. y Trejo, I. (2010). Plant propagation and the ecological restoration of Mexican tropical deciduous forests. Ecological Restoration, 28, 369-376.

Bradshaw, A. D. (1987). The reclamation of derilict land and the ecology of ecosystems. En W. R. Jordan, M. E. Gilpin, y J. D. Aber (Eds.), Restoration ecology: a synthetic approach to ecological research (pp. 53-74). Cambridge: Cambridge University Press.

Bullock, J. M., Aronson, J., Newton, A. C., Pywell, R. F. y Rey-Benayas, J. M. (2011). Restoration of ecosystem services and biodiversity: conflicts and opportunities. Trends in Ecology \& Evolution, 26, 541-549.

Campbell, W. B., Freeman, D. C., Emlen, J. M. y López-Ortiz, S. (2010). Correlations between plant phylogenetic and functional diversity in a high altitude cold salt desert depend on sheep grazing season: implications for range recovery. Ecological Indicators, 10, 676-686.

Cantarello, E., Newton, A. C., Hill, R. A., Tejedor-Garavito, N., WilliamsLinera, G., López-Barrera, F., et al. (2011). Simulating the potential for ecological restoration of dryland forests in Mexico under different disturbance regimes. Ecological Modelling, 222, 1112-1128.

Carabias, J., Arriaga, V. y Gutiérrez, V. (2007). Las políticas públicas de la restauración ambiental en México: limitantes, avances, rezagos y retos. Boletín de la Sociedad Botánica de México, 80, 85-100.

Carabias, J., Ruíz, L. y Rabasa, A. (2016). El marco legal de la restauración de ecosistemas forestales en México. En E. Ceccon y C. Martínez-Garza (Eds.), Experiencias mexicanas en la restauración de los ecosistemas (pp. 37-74). Cuernavaca: Conabio, CRIM-UNAM, UAEM.

Ceccon, E. (2013). Restauración en bosques tropicales: fundamentos ecológicos, prácticos y sociales. México D.F.: CRIM-UNAM/Ediciones Díaz de Santos.

Ceccon, E., Barrera-Cataño, J. I., Aronson, J. y Martínez-Garza, C. (2015). The socioecological complexity of ecological restoration in Mexico. Restoration Ecology, 23, 331-336

Ceccon, E., González, E. J. y Martorell, C. (2015). Is direct seeding a biologically viable strategy for restoring forest ecosystems? Evidences from a metaanalysis. Land Degradation \& Development, 27, 511-520.

Ceccon, E., y Martínez-Garza, C. (Eds.). (2016). Experiencias mexicanas en la restauración de los ecosistemas. Cuernavaca: Conabio, CRIM-UNAM, UAEM.

Ceccon, E., y Pérez, D. R. (Eds.). (2016). Más allá de la ecología de la restauración: perspectivas sociales en América Latina y el Caribe. Buenos Aires: Vázquez Mancini,

Ceccon, E., Sánchez, I. y Powers, J. (2014). Biological potential of four indigenous tree species from seasonally dry tropical forest for soil restoration. Agroforestry Systems, 89, 455-467.

Challenger, A., Dirzo, R., López, J. C., Mendoza, E. y Lira-Noriega, A. (2009). Factores de cambio y estado de la biodiversidad. En J. Sarukhán (Ed.), Capital natural de México. Vol. II: Estado de conservación y tendencias de cambio (pp. 37-73). México D.F.: Conabio.

Cirelli, V. y Sánchez-Cordero, V. (2009). Selection of restoration and conservation areas using species ecological niche modeling: a case study of the Neotropical river otter Lontra longicaudis annectens in central Mexico. En A. Columbus y L. Kuznetsov (Eds.), Endangered species: new research (pp. 261-278). New York: Nova Science Publishers.

CNA (Comisión Nacional del Agua) (2013). Estadísticas del agua en México [consultado Jun 2015]. Disponible en: http://www.conagua. gob.mx/CONAGUA07/Noticias/SGP-2-14Web.pdf

Conabio (Comisión Nacional para el Conocimiento y Uso de la Biodiversidad) (2009). Capital natural de México. Vol. II: Estado de conservación y tendencias de cambio. México D.F.: Conabio. 
Conabio (Comisión Nacional para el Conocimiento y Uso de la Biodiversidad) (2012). Estrategia mexicana para la conservación vegetal, 2012-2030. México D.F.: Conabio.

Conabio (Comisión Nacional para el Conocimiento y Uso de la Biodiversidad) (2014). Quinto Informe Nacional de México ante el Convenio sobre la Diversidad Biológica $(C D B)$. México D.F.: Conabio.

Conafor (Comisión Nacional Forestal) (2014). Recuperación de superficie forestal mediante reforestación. Revista Innovación Forestal [consultado Jun 2015]. Disponible en: http://www.conafor.gob.mx/ innovacion_forestal/?p=1009

De la Peña-Domene, M., Martínez-Garza, C., Palmas-Pérez, S., Rivas-Alonso, E. y Howe, H. F. (2014). Roles of birds and bats in early tropical-forest restoration. Plos One, 9, e104656.

De la Rosa-Mera, C. J. y Monroy-Ata, A. (2006). Plant assembly for ecological restoration in a semiarid zone. TIP Revista Especializada en Ciencias Químico-Biológicas, 9, 96-100.

Dhillion, S. S., Aguilar-Stoen, M. y Camargo-Ricalde, S. L. (2004). Integrative ecological restoration and the involvement of local communities in the Tehuacán-Cuicatlán Valley, Mexico. Environmental Conservation, 31, $1-3$.

Díaz-Rodríguez, B., Blanco-García, A., Gómez-Romero, M. y Lindig-Cisneros, R. (2012). Filling the gap: restoration of biodiversity for conservation in productive forest landscapes. Ecological Engineering, 40, 88-94.

Diemont, S. A. W., Martin, J. F., Levy-Tacher, S. I., Nigh, R. B., López, P. R. y Golicher, J. D. (2006). Lacandon Maya forest management: restoration of soil fertility using native tree species. Ecological Engineering, 28, $205-212$.

Domínguez, C., Bojórquez, L., Boege, K., Fornoni, J., Gómez, P., Valiente, A., et al. (2009). Sinergias entre el cambio climático y las especies exóticas invasoras. México D.F.: Instituto Nacional de Ecología y Cambio Climático e Instituto de Ecología.

Douterlungne, D., Levy-Tacher, S. I., Golicher, D. J. y Roman-Danobeytia, F. (2008). Applying indigenous knowledge to the restoration of degraded tropical rain forest clearings dominated by bracken fern. Restoration Ecology, $18,322-329$.

Douterlungne, D. y Ferguson, B. G. (2012). Manual de restauración ecológica campesina para la selva Lacandona. Guadalajara: ECOSUR, Fondo Conacyt-Conafor.

Douterlungne, D., Thomas, E. y Levy-Tacher, S. I. (2013). Fast-growing pioneer tree stands as a rapid and effective strategy for bracken elimination in the Neotropics. Journal of Applied Ecology, 50, 1257-1265.

Encino-Ruiz, L., Lindig-Cisneros, R., Gómez-Romero, M. y Blanco-García, A. (2013). Performance of three tropical forest tree species in a test deciduous ecological restoration. Botanical Sciences, 91, 107-114.

FAO (Food and Agriculture Organization) (2010). Evaluación de los recursos forestales mundiales [consultado Jun 2015]. Disponible en: http://www.fao.org/docrep/013/i1757s/i1757s.pdf

FAO (Food and Agriculture Organization) (2015a). Evaluación de recursos forestales mundiales. Compendio de datos [consultado Jun 2015]. Disponible en: http://www.fao.org/3/a-i4808s.pdf

FAO (Food and Agriculture Organization) (2015b). Evaluación de los recursos forestales mundiales. Informe nacional, México [consultado Jun 2015]. Disponible en http://www.fao.org/3/a-az275s.pdf

Flores-Verdugo, F., Moreno-Casasola, P., Agraz-Hernández, C. M., LópezRosas, H., Benítez-Pardo, D. y Travieso-Bello, A. C. (2007). La topografía y el hidroperíodo: dos factores que condicionan la restauración de los humedales costeros. Boletín de la Sociedad Botánica de México, 80, 33-47.

Foroughbakhch, R., Alvarado-Vázquez, M. A., Hernández-Pinero, J. L., Rocha-Estrada, A., Guzmán-Lucio, M. A. y Treviño-Garza, E. J. (2006) Establishment, growth and biomass production of 10 tree woody species introduced for reforestation and ecological restoration in Northeastern Mexico. Forest Ecology and Management, 235, 194-201.

Fragoso, C. y Rojas-Fernández, P. (2012). Monitoreo ecológico de una cantera rehabilitada por cementos Holcim Apasco en Veracruz. Xalapa: INECOL y Holcim Apasco.

Fuentealba, B. D. y Martínez-Ramos, M. (2014). Transplanting native tree seedlings to enrich tropical live fences: an ecological and socio-economic analysis. Agroforestry Systems, 88, 221-236.
Gaze, L. y Breen, J. (2014). The research \& innovation performance of the G20. Thomson Reuters [consultado Jun 2016]. Disponible en: http://sciencewatch. $\mathrm{com} / \mathrm{sites} / \mathrm{sw} /$ files/images/basic/research-innovation-g20.pdf.

Gelviz-Gelvez, S. M., Pavón, N. P., Illoldi-Rangel, P. y Ballesteros-Barrera, C. (2015). Ecological niche modeling under climate change to select shrubs for ecological restoration in Central Mexico. Ecological Engineering, 74, 302-309.

Gómez-Pompa, A. (1973). Ecology of the vegetation of Veracruz. En A. Graham (Ed.), Vegetation and vegetational history of Northern Latin America (pp. 73-148). Amsterdam: Elsevier Scientific.

Gómez-Pompa, A. y del Amo, S. (1985). Investigaciones sobre la regeneración de selvas altas en Veracruz, México. México D.F.: Alhambra Mexicana S.A. de C.V.

Gómez-Pompa, A., del Amo, S., Vázquez-Yanes, C. y Butanda, C. A. (1976). Investigaciones sobre la regeneración de selvas altas en Veracruz. México D.F.: CECSA.

Gómez-Pompa, A. y Vázquez-Yanes, C. (1981). Successional studies of a rain forest in México. En D. C. West, H. H. Shugart, y D. B. Butkin (Eds.), Forest succession concepts and application (pp. 246-266). New York: SpringerVerlag.

González-Espinosa, M., Ramírez-Marcial, N., Camacho-Cruz, A., Holz, S. C., Rey-Benayas, J. M. y Vázquez, M. R. P. (2007). Restauración de bosques en territorios indígenas de Chiapas: modelos ecológicos y estrategias de acción. Boletín de la Sociedad Botánica de México, 80, 11-23.

González-Espinoza, M., Rey-Benayas, J. M. y Ramírez-Marcial, N. (2008). Restauración de bosques en América Latina. Fundación Internacional para la Restauración de Ecosistemas. México D.F.: Mundi-Prensa.

González-Ovando, M. L., Plascencia-Escalante, F. O. y Martínez-Trinidad, T. (2016). Áreas prioritarias para restauración ecológica y sitios de referencia en la región Chignahuapan-Zacatlán. Madera y Bosques, 22, 41-52.

González-Reynoso, G. A., Hernández-Muñoz, L. M., Perló, C. M. y Zamora, S. I. (2010). Rescate de ríos urbanos: propuestas conceptuales y metodológicas para restauración y rehabilitación de ríos. México D.F.: Coordinación de Humanidades. Programa Universitario de Estudios sobre la Ciudad. Universidad Nacional Autónoma de México.

González-Tokman, D. y Martínez-Garza, C. (2015). Effect of ecological restoration on body condition of a predator. Plos One, 10, e0133551.

Guevara, S., Purata, S. E. y van der Maarel, E. (1986). The role of remnant forest trees in tropical secondary succession. Vegetatio, 66, 77-84.

Hansen, A. M., van Afferden, M. y Torres-Beiarano, F. (2007). Remediation of the Cencali lagoon, Villahermosa, Tabasco. I. Pollution and sediment reuse. Ingeniería Hidráulica en México, 22, 87-102.

Hernández, Y., Boege, K., Lindig-Cisneros, R. y del Val, E. (2014). Lepidopteran herbivory in restored and successional sites in a tropical dry forest. The Southwestern Naturalist, 59, 66-74.

Hernández-Carmona, G., García, O., Robledo, D. y Foster, M. (2000). Restoration techniques for Macrocystis pyrifera (Phaeophyceae) populations at the southern limit of their distribution in Mexico. Botánica Marina, 43, 273-284.

Hernández-Flores, J., Osorio-Beristain, M. y Martínez-Garza, C. (2016). Ant foraging as an indicator of Tropical Dry Forest restoration. Environmental Entomology, 45, 991-994.

Hernández-Ladrón de Guevara, I., Rojas-Soto, O. R., López-Barrera, F., PueblaOlivares, F. y Díaz-Castelazo, C. (2012). Seed dispersal by birds in a cloud forest landscape in central Veracruz, Mexico: its role in passive restoration. Revista Chilena de Historia Natural, 85, 89-100.

Hernández-Xolocotzi, E. (1988). La agricultura tradicional en México. Comercio Exterior, 38, 673-678.

Holguin, G., Vázquez, P. y Bashan, Y. (2001). The role of sediment microorganisms in the productivity, conservation, and rehabilitation of mangrove ecosystems: an overview. Biology and Fertility of Soils, 33, 265-278.

Howe, H. y Martínez-Garza, C. (2014). Restauración experimental. Botanical Sciences, 92, 459-468.

Huante, P., Ceccon, E., Orozco-Segovia, A., Sánchez-Coronado, M. E., Acosta, I. y Rincón, E. (2012). The role of arbuscular mycorrhizal fungi on the early stage restoration of seasonally dry tropical forest in Chamela, Mexico. Revista Arvore, 36, 279-289.

Islas-Peña, T. V., Liñán-Cabello, M. A. y Torres-Orozco, E. (2004). Evaluation of two techniques for restoration of Pocillopora sp.: early effects of species and 
marine environment. En M. A. Liñán-Cabello (Ed.), Corals: classification, habitat and ecological significance (pp. 133-152). New York: Nova Science Publishers.

IUCN (International Union for Conservation of Nature) (2014). Países de Latinoamérica y el Caribe lanzan la iniciativa $20 \times 20$ para restaurar 20 millones de hectáreas de tierra degradada [consultado Jun 2015]. Disponible en http://www.iucn.org/es/?18699/20-20-cop-20

Jiménez, C., Huante, P. y Rincón, E. (2006). Restauración de minas superficiales en México. México D.F.: Instituto de Ecología, UNAM.

Jiménez, J., Jurado, E., Aguirre, O. y Estrada, E. (2005). Effect of grazing on restoration of endemic dwarf pine (Pinus culminicola Andresen et Beaman) populations in Northeastern Mexico. Restoration Ecology, 13, $103-107$.

Juan-Baeza, I., Martínez-Garza, C. y del Val, E. (2015). Recovering more than tree cover: herbivores and herbivory in a restored Tropical Dry Forest. Plos One, 10, e0128583.

Landgrave, R. y Moreno-Casasola, P. (2012). Evaluación cuantitativa de la pérdida de humedales en México. Investigación Ambiental, 4, 19-35.

Lara-Cabrera, S. I., Alejandre-Melena, N., Medina-Sánchez, E. I. y LindigCisneros, R. (2009). Genetic diversity in populations of Lupinus elegans kunth, implications for ecological restoration. Revista Fitotecnia Mexicana, 32, 79-86.

Latofski-Robles, M., Aguirre-Muñoz, A., Méndez-Sánchez, F., ReyesHernández, H. y Schlüter, S. (2014). Prioritizing restoration actions for the islands of Mexico. Monographs of the Western North American Naturalist, 7, 435-441.

Lindig-Cisneros, R., Blanco-García, A. y Sáenz-Romero, C. (2007). Restauración adaptable en la meseta Purépecha, Michoacán, México: hacia un modelo de estados y transiciones. Boletín de la Sociedad Botánica de México, 80, 25-31.

Lindig-Cisneros, R., Desmond, J., Boyer, K. E. y Zedler, J. B. (2003). Wetland restoration thresholds: Can a degradation transition be reversed with increased effort? Ecological Applications, 13, 193-205.

Liñan-Cabello, M. A., Flores-Ramírez, L. A., Laurel-Sandoval, M. A., GarcíaMendoza, E., Soriano-Santiago, O. y Delgadillo-Nuño, M. A. (2011). Acclimation in Pocillopora spp. during a coral restoration program in Carrizales Bay, Colima, Mexico. Marine and Freshwater Behaviour and Physiology, 44, 61-72.

Lithgow, D., Martínez, M. L. y Gallego-Fernández, J. B. (2015). The "ReDune" index (Restoration of coastal Dunes Index) to assess the need and viability of coastal dune restoration. Ecological Indicators, 49, 178-187.

Lithgow, D., Martínez, M. L., Gallego-Fernández, J. B., Hesp, P. A., Flores, P., Gachuz, S., et al. (2013). Linking restoration ecology with coastal dune restoration. Geomorphology, 199, 214-224.

López-Lozano, N. E., Carcaño-Montiel, M. G. y Bashan, Y. (2016). Using native trees and cacti to improve soil potential nitrogen fixation during long-term restoration of arid lands. Plant and Soil, 403, 317-329.

López-Rosas, H., López-Barrera, F., Moreno-Casasola, P., Aguirre-León, G., Cázares-Hernández, E. y Sánchez-Higueredo, L. (2010). Indicators of recovery in a tropical freshwater marsh invaded by an African grass. Ecological Restoration, 28, 324-332.

MacGregor-Fors, I., Blanco-García, A. y Lindig-Cisneros, R. (2010). Bird community shifts related to different forest restoration efforts: a case study from a managed habitat matrix in Mexico. Ecological Engineering, 36, 1492-1496.

MAE (Ministerio del Ambiente de Ecuador) (2014). Plan Nacional de Restauración Forestal 2014-2017, Ministerio de Medio Ambiente, Quito, Ecuador. 50 p. [consultado Jun 2015]. Disponible en: http://www.ambiente. gob.ec/tag/plan-nacional-de-restauracion-forestal/

Martínez, L. M., Gallego-Fernández, J. B. y Hesp, P. A. (2013). Restoration of coastal dunes. Berlin, Heidelberg: Springer-Verlag.

Martínez, M. L. y López-Barrera, F. (2008). Special issue: restoring and designing ecosystems for a crowded planet. Ecoscience, 15, 1-5.

Martínez, M. L., Moreno-Casasola, P., Espejel, I., Jiménez-Orocio, O., InfanteMata, D. y Rodríguez-Revelo, N. (2014). Diagnóstico general de las dunas costeras de México. Ciudad de México: Comisión Nacional Forestal.

Martínez-Garza, C., Bongers, F. y Poorter, L. (2013). Are functional traits good predictors of species performance in restoration plantings in tropical abandoned pastures? Forest Ecology and Management, 303, 35-45.
Martínez-Garza, C., Peña, V., Ricker, M., Campos, A. y Howe, H. F. (2005). Restoring tropical biodiversity: leaf traits predict growth and survival of late-successional trees in early-successional environments. Forest Ecology and Management, 217, 365-379.

Mazari-Hiriart, M., Pérez-Ortiz, G., Orta-Ledesma, M. T., Armas-Vargas, F., Tapia, M. A., Solano-Ortiz, R., et al. (2014). Final opportunity to rehabilitate an urban river as a water source for Mexico City. Plos One, 9, e102081.

Martínez-Ramos, M. y García-Orth, X. (2007). Sucesión ecológica y restauración de las selvas húmedas. Boletín de la Sociedad Botánica de México, 80 69-84.

Meli, P. y Carrasco-Carballido, V. (2011). Restauración ecológica de riberas: manual para la recuperación de la vegetación ribereña en arroyos de la selva Lacandona, México. Ciudad de México: Corredor Biológico Mesoamericano México, Comisión Nacional para el Conocimiento y Uso de la Biodiversidad

Meli, P. y Dirzo, R. (2012). Effects of grasses on sapling establishment and the role of transplanted saplings on the light environment of pastures: implications for tropical forest restoration. Applied Vegetation Science, 16, 296-304.

Meli, P., Martínez-Ramos, M. y Rey-Benayas, J. M. (2013). Selecting species for passive and active riparian restoration in Southern Mexico. Restoration Ecology, 21, 163-165.

Meli, P., Martínez-Ramos, M., Rey-Benayas, J. M. y Carabias, J. (2014). Combining ecological, social and technical criteria to select species for forest restoration. Applied Vegetation Science, 17, 744-753.

Meli, P., Rey-Benayas, J. M., Balvanera, P. y Martínez-Ramos, M. (2014). Restoration enhances wetland biodiversity and ecosystem service supply, but results are context-dependent: a meta-analysis. Plos One, 9, e93507.

Mendoza-Bautista, C., García-Moreno, F. y Rodríguez-Trejo, D. A. (2010). Restoring the Vara de Perlilla in La Mesa, México. Ecological Restoration, 28, 262-263.

Mendoza-Hernández, P. E. y Cano-Santana, Z. (2009). Elementos para la restauración ecológica de pedregales: la rehabilitación de áreas verdes de la Facultad de Ciencias en Ciudad Universitaria. En A. Lot y Z. CanoSantana (Eds.), Biodiversidad del ecosistema del Pedregal de San Ángel (pp. 523-532). Ciudad de México: UNAM.

Mendoza-Hernández, P. E., Orozco-Segovia, A. y Pisanty, I. (2010). Germination, emergence, and survival of Buddleja cordata in an urban forest. Ecological Restoration, 28, 263-265.

Mendoza-Hernández, P. E., Orozco-Segovia, A., Meave, J. A., Valverde, T. y Martínez-Ramos, M. (2013). Vegetation recovery and plant facilitation in a human-disturbed lava field in a megacity: searching tools for ecosystem restoration. Plant Ecology, 214, 153-167.

Miranda, F. y Hernández-Xolocotzi, E. (1963). Los tipos de vegetación de México y su clasificación. Boletín de la Sociedad Botánica de México, 28, 29-179.

Monroy-Ata, A., Estévez-Torres, J., García-Sánchez, R. y Ríos-Gómez, R. (2007). Establecimiento de plantas mediante el uso de micorrizas y de islas de recursos en un matorral xerófilo deteriorado. Boletín de la Sociedad Botánica de México, 80, 49-57.

Morales-Barquero, L., Skutsch, M., Jardel-Peláez, E. J., Ghilardi, A., Kleinn, C. y Healey, J. R. (2014). Operationalizing the definition of forest degradation for REDD+, with application to Mexico. Forests, 5, 1653-1681.

Moreno-Casasola, P., Infante, D. M., Travieso-Bello, A. C. y Madero-Vega, C. (2009). Manual para la reforestación de los médanos. Xalapa: Instituto de Ecología A.C., Conafor, Conacyt.

Moreno-Casasola, P., Martínez, M. L. y Castillo-Campos, G. (2008). Designing ecosystems in degraded tropical coastal dunes. Ecoscience, 15, 44-52.

Murcia, C. y Guariguata, M. R. (2014). La restauración ecológica en Colombia: tendencias, necesidades y oportunidades [CIFOR Occasional paper no. 107]. Bogor, Indonesia: Center for International Forestry Research (CIFOR).

Murgueitio, E., Calle, Z., Uribe, F., Calle, A. y Solorio, B. (2011). Native trees and shrubs for the productive rehabilitation of tropical cattle ranching lands. Forest Ecology and Management, 261, 1654-1663.

Newton, A. C., del Castillo, R. F., Echeverría, C., Geneletti, D., GonzálezEspinosa, M., Malizia, L. R., et al. (2012). Forest landscape restoration in the drylands of Latin America. Ecology and Society, 17, 21. 
Niembro, A., Sánchez, O. y Vásquez, M. (2010). Árboles de Veracruz: 100 especies para la reforestación estratégica. Ciudad de México: Gobierno del estado de Veracruz.

Ochoa-Ochoa, L. M., Rodríguez, P., Mora, F., Flores-Villela, O. y Whittaker, R. J. (2012). Climate change and amphibian diversity patterns in Mexico. Biological Conservation, 150, 94-102.

Olguín, E. J., Hernández, M. E. y Sánchez-Galván, G. (2007). Hydrocarbon mangroves pollution and bioremediation, phytoremediation and restoration strategies. Revista Internacional de Contaminación Ambiental, 23, 139-154.

Oyama, K., y Castillo, A. (Eds.). (2006). Manejo, conservación y restauración de recursos naturales en México: perspectivas desde la investigación científica. México D.F.: Universidad Nacional Autónoma de México, Centro de Investigaciones en Ecosistemas, Siglo XXI Editores.

Palmer, M. A. (2009). Reforming watershed restoration: science in need of application and applications in need of science. Estuaries and Coasts, 32, $1-17$.

Purata, S. E. (1986a). Transect analysis a basis for comparing of old-field succession in a tropical rain forest area in Mexico. Tropical Ecology, 27, $103-122$.

Purata, S. E. (1986b). Floristic and structural changes during old-field succession in the Mexican tropics in relation to site history and species availability. Journal of Tropical Ecology, 2, 257-276.

Ramírez-Hernández, J., Hinojosa-Huerta, O., Peregrina-Llanes, M., CalvoFonseca, A. y Carrera-Villa, E. (2013). Groundwater responses to controlled water releases in the limitrophe region of the Colorado River: implications for management and restoration. Ecological Engineering, 59, 93-103.

Ramírez-Herrejón, J. P., Zambrano, L., Mercado-Silva, N., Torres-Téllez, A., Pineda-García, F., Caraveo-Patiño, J., et al. (2014). Long term changes in the fish fauna of Lago de Pátzcuaro in Central Mexico. Latin American Journal of Aquatic Research, 42, 137-149.

Ramírez-Marcial, N., González-Espinosa, M., Camacho-Cruz, A. y OrtizAguilar, D. (2010). Forest restoration in Lagunas de Montebello National Park, Chiapas, Mexico. Ecological Restoration, 28, 354-360.

Ramírez-Marcial, N., González-Espinosa, M., Musálem-Castillejos, K., Savelli, E. N. y Gómez-Pineda, E. (2014). Estrategias para una construcción social de la restauración forestal en comunidades de la cuenca media y alta del río Grijalva. En M. González-Espinosa y M. C. Brunel (Eds.), Montañas, pueblos y agua: dimensiones y realidades de la cuenca Grijalva (pp. 518-554). Ciudad de México: Editorial Juan Pablos.

Ramírez-Marcial, N., Luna-Gómez, A., Castañeda-Ocaña, H. E., Martínez-Icó, M., Holz, S., Camacho-Cruz, A., et al. (2012). Guía de propagación de árboles nativos para la recuperación de bosques. San Cristóbal de Las Casas: ECOSUR.

Reyes-Chargoy, M. A. y Tovilla-Hernández, C. (2002). Restauración de áreas alteradas de manglar con Rhizophora mangle en la Costa de Chiapas. Madera y Bosques, 8, 103-114.

Rodríguez-Trejo, D. A. y Myers, R. L. (2010). Using oak characteristics to guide fire regime restoration in Mexican pine-oak and oak forests. Ecological Restoration, 28, 304-323.

Rodríguez-Zúñiga, M. T., Troche-Souza, C., Vázquez-Lule, A. D., MárquezMendoza, J. D., Vázquez-Balderas, B., Valderrama-Landeros, L., et al. (2013). Manglares de México: extensión, distribución y monitoreo. Ciudad de México: Conabio.

Roman-Danobeytia, F. J., Levy-Tacher, S. I., Aronson, J., Rodrigues, R. R. y Castellanos-Albores, J. (2012). Testing the performance of fourteen native tropical tree species in two abandoned pastures of the Lacandon Rainforest region of Chiapas, Mexico. Restoration Ecology, 20, 378-386.

Salinas-Rodríguez, A. S. y Ramírez-Marcial, N. (2010). "El Triunfo" biosphere reserve rivers, a diagnosis for their hydrological restoration. Hidrobiologica, 20, 91-100.

San José, M., Garmendia, A. y Cano-Santana, Z. (2013). Vertebrate fauna evaluation after habitat restoration in a Reserve within Mexico City. Ecological Restoration, 31, 249-252.

Sánchez, O., Peters, E., Vega, E., Márquez-Huitzil, R., Portales, G., Valdez, M., et al. (2005). Temas sobre restauración ecológica. México, D.F.: Instituto Nacional de Ecología.

Santos-del Prado, K. y Peters, E. (2008). Isla Guadalupe: restauración y conservación. México, D.F.: Instituto Nacional de Ecología.
Sarukhán, J., Urquiza-Haas, T., Koleff, P., Carabias, J., Dirzo, R., Ezcurra, E., et al. (2015). Strategic actions to value, conserve, and restore the natural capital of megadiversity countries: the case of Mexico. BioScience, 65, 164-173.

Sedatu (Secretaría de Desarrollo Agrario, Territorial y Urbano) (2013). Programa sectorial de desarrollo agrario, territorial y urbano 2013-2018 [consultado Jun 2015]. Disponible en: http://www.dof. gob.mx/nota_detalle.php?codigo $=5326473 \&$ fecha $=16 / 12 / 2013$

SER (Society for Ecological Restoration International Science \& Policy Working Group) (2004). The SER International primer on ecological restoration. Washington D.C. [consultado Jun 2015]. Disponible en: http:// www.ser.org/resources/resources-detail-view/ser-international-primer-onecological-restoration

Suárez, A., Williams-Linera, G., Trejo, C., Valdez-Hernández, J. I., CetinaAlcalá, V. M. y Vibrans, H. (2012). Local knowledge helps select species for forest restoration in a tropical dry forest of central Veracruz, Mexico. Agroforestry Systems, 85, 35-55.

Suárez-Guerrero, A. I. y Equihua, M. (2005). Experimental tree assemblages on the ecological rehabilitation of a cloud forest in Veracruz, Mexico. Forest Ecology and Management, 218, 329-341.

Tobón, W., Koleff, P., Urquiza-Haas, T. y García-Méndez, G. (2016). Propuesta metodológica para identificar prioridades de restauración en México. En E. Ceccon y C. Martínez-Garza (Eds.), Experiencias mexicanas en la restauración de los ecosistemas (pp. 18-36). Cuernavaca: Conabio, CRIM-UNAM, UAEM

Toledo, G., Rojas, A. y Bashan, Y. (2001). Monitoring of black mangrove restoration with nursery-reared seedlings on an arid coastal lagoon. Hydrobiologia, 444, 101-109.

Tortolero-Langarica, J. J. A., Cupul-Magaña, A. L. y Rodríguez-Troncoso, A. P. (2014). Restoration of a degraded coral reef using a natural remediation process: a case study from a Central Mexican Pacific National Park. Ocean and Coastal Management, 96, 12-19.

Trabucchi, M., Puente, C., Comin, F. A., Olague, G. y Smith, S. V. (2012). Mapping erosion risk at the basin scale in a Mediterranean environment with opencast coal mines to target restoration actions. Regional Environmental Change, 12, 675-687.

Ureta, C. y Martorell, C. (2009). Identifying the impacts of chronic anthropogenic disturbance on two threatened cacti to provide guidelines for population-dynamics restoration. Biological Conservation, 142, 1992-2001

Uribe, D., Geneletti, D., del Castillo, R. F. y Orsi, F. (2014). Integrating stakeholder preferences and gis-based multicriteria analysis to identify forest landscape restoration priorities. Sustainability, 6, 935-951.

Urquía-Fernández, N. (2014). Food security in Mexico. Salud Pública de México, 56, 92-98.

Valiente, E., Tovar, A., González, H., Eslava-Sandoval, D. y Zambrano, L. (2010). Creating refuges for the axolotl (Ambystoma mexicanum). Ecological Restoration, 28, 257-259.

Vázquez-Yanes, C. y Batis, A. I. (1996). Domestication of wild native plants for ecological restoration and reforestation. Boletín de la Sociedad Botánica de México, 75-84.

Verdú, M., Gómez-Aparicio, L. y Valiente-Banuet, A. (2012). Phylogenetic relatedness as a tool in restoration ecology: a meta-analysis. Proceedings of the Royal Society B: Biological Sciences, 279, 1761-1767.

Villa-Galaviz, E., Boege, K. y del Val, E. (2012). Resilience in plant-herbivore networks during secondary succession. Plos One, 7, e53009.

Von Bertrab, A. y Zambrano, L. (2010). Participatory monitoring and evaluation of a Mexico City wetland restoration effort. Ecological Restoration, 28, 343-353.

Vovides, A. G., Bashan, Y., López-Portillo, J. A. y Guevara, R. (2011). Nitrogen fixation in preserved, reforested, naturally regenerated and impaired mangroves as an indicator of functional restoration in mangroves in an arid region of Mexico. Restoration Ecology, 19, 236-244.

West, J. D., Jacquet, J., King, M. M., Correll, S. J. y Bergstrom, C. T. (2013). The role of gender in scholarly authorship. Plos One, 8, e66212.

Williams-Linera, G. y Álvarez-Aquino, C. (2010). Tropical dry forest landscape restoration in Central Veracruz, Mexico. Ecological Restoration, 28, 259-261. 
Williams-Linera, G., López-Barrera, F. y Bonilla-Moheno, M. (2015). Establishing the baseline for cloud forest restoration in a peri-urban landscape. Madera y Bosques, 21, 89-101.

Yáñez-Arancibia, A., Day, J. W., Sánchez-Gil, P., Day, J. N., Lane, R. R., ZárateLomeli, D., et al. (2014). Ecosystem functioning: the basis for restoration and management of a tropical coastal lagoon, Pacific coast of Mexico. Ecological Engineering, 65, 88-100.

Young, T. P., Petersen, D. A. y Clary, J. J. (2005). The ecology of restoration: historical links, emerging issues and unexplored realms. Ecology Letters, 8, $662-673$.
Zaldívar-Jiménez, M. A., Herrera-Silveira, J. A., Teutli-Hernández, C., Comín, F. A., Andrade, J. L., Molina, C. C., et al. (2010). Conceptual framework for mangrove restoration in the Yucatán Peninsula. Ecological Restoration, 28, 333-342.

Zepeda-Gómez, C., Lot, A., Antonio-Nemiga, X. y Manjarrez, J. (2015) Evaluation of the seed banks and its importance in the rehabilitation of the wetland vegetation of Central Mexico. Botanical Sciences, 93, 695-707. 